\title{
Existence of global strong solutions in critical spaces for barotropic viscous fluids
}

\author{
Boris Haspot ${ }^{* \dagger}$
}

\begin{abstract}
This paper is dedicated to the study of viscous compressible barotropic fluids in dimension $N \geq 2$. We address the question of the global existence of strong solutions for initial data close to a constant state having critical Besov regularity. First, this article shows the recent results of [7] and [11] with a new proof. Our result relies on a new a priori estimate for the velocity that we derive via the intermediary of the effective velocity, which allows us to cancel out the coupling between the density and the velocity as in [21]. Second we improve the results of [7] and [11] by adding as in [7] some regularity on the initial data in low frequencies. In this case we obtain global strong solutions for a class of large initial data which rely the results of D. Hoff in $[25,28,29]$ and those of $[7,11]$. We conclude by generalizing these results for general viscosity coefficients.
\end{abstract}

\section{Introduction}

The motion of a general barotropic compressible fluid is described by the following system:

$$
\left\{\begin{array}{l}
\partial_{t} \rho+\operatorname{div}(\rho u)=0 \\
\partial_{t}(\rho u)+\operatorname{div}(\rho u \otimes u)-\operatorname{div}(\mu(\rho) D(u))-\nabla(\lambda(\rho) \operatorname{div} u)+\nabla P(\rho)=\rho f \\
(\rho, u)_{/ t=0}=\left(\rho_{0}, u_{0}\right) .
\end{array}\right.
$$

Here $u=u(t, x) \in \mathbb{R}^{N}$ stands for the velocity field and $\rho=\rho(t, x) \in \mathbb{R}^{+}$is the density. The pressure $P$ is a suitable smooth function of $\rho$. We denote by $\lambda$ and $\mu$ the two viscosity coefficients of the fluid, which are assumed to satisfy $\mu>0$ and $\lambda+2 \mu>0$ (in the sequel to simplify the calculus we will assume the viscosity coefficients as constants). Such a condition ensures ellipticity for the momentum equation and is satisfied in the physical cases where $\lambda+\frac{2 \mu}{N}>0$. We supplement the problem with initial condition $\left(\rho_{0}, u_{0}\right)$ and an outer force $f$. Throughout the paper, we assume that the space variable $x \in \mathbb{R}^{N}$ or to the periodic box $\mathcal{T}_{a}^{N}$ with period $a_{i}$, in the i-th direction. We restrict ourselves to the case $N \geq 2$.

The problem of existence of global solutions for Navier-Stokes equations was addressed in one dimension for smooth enough data by Kazhikov and Shelukin in [33], and for

\footnotetext{
* Karls Ruprecht Universität Heidelberg, Institut for Applied Mathematics, Im Neuenheimer Feld 294, D-69120 Heildelberg, Germany. Tel. 49(0)6221-54-6112

${ }^{\dagger}$ Basque Center of Applied Mathematics, Bizkaia Technology Park, Building 500, E-48160, Derio (Spain), haspot@bcamath.org
} 
discontinuous ones, but still with densities away from zero, by Serre in [40] and Hoff in [23]. Those results have been generalized to higher dimension by Hoff in $[26,27]$. The existence and uniqueness of local classical solutions for (1.1) with smooth initial data such that the density $\rho_{0}$ is bounded and bounded away from zero has been stated by Nash in [38]. Let us emphasize that no stability condition was required there. On the other hand, for small smooth perturbations of a stable equilibrium with constant positive density, global well-posedness has been proved in [36]. More precisely Matsumura and Nishida in [36] obtained the existence of global strong solutions for three-dimensional polytropic ideal fluids and no outer force with initial data chosen small in the following spaces $\left(\rho_{0}-1, u_{0}\right) \in H^{3} \times H^{3}$. Refined functional analysis has been used for the last decades, ranging from Sobolev, Besov, Lorentz and Triebel spaces to describe the regularity and long time behavior of solutions to the compressible model [41], [42], [25], [32].

Guided in our approach by numerous works dedicated to the incompressible Navier-Stokes equation (see e.g [37]):

$$
\left\{\begin{array}{l}
\partial_{t} v+v \cdot \nabla v-\mu \Delta v+\nabla \Pi=0, \\
\operatorname{div} v=0
\end{array}\right.
$$

we aim at solving (1.1) in the case where the data $\left(\rho_{0}, u_{0}, f\right)$ have critical regularity.

By critical, we mean that we want to solve the system (1.1) in functional spaces with norm invariant by the changes of scales which leave (1.1) invariant. In the case of barotropic fluids, it is easy to see that the transformations:

$$
(\rho(t, x), u(t, x)) \longrightarrow\left(\rho\left(l^{2} t, l x\right), l u\left(l^{2} t, l x\right)\right), \quad l \in \mathbb{R},
$$

have that property, provided that the pressure term has been changed accordingly.

Definition 1.1 Let $\bar{\rho}>0$. In the sequel we will note: $q=\frac{\rho-\bar{\rho}}{\bar{\rho}}$.

The use of critical functional frameworks led to several new well-posedness results for compressible fluids (see $[13,17,19,21]$ ). In addition to have a norm invariant by $(1.2)$, appropriate functional spaces for solving (1.1) must provide a control on the $L^{\infty}$ norm of the density (in order to avoid vacuum and loss of ellipticity). For that reason, we restricted our study to the case where the initial data $\left(\rho_{0}, u_{0}\right)$ and external force $f$ are in homogeneous Besov spaces such that, for some positive constant $\bar{\rho}$ :

$$
q_{0} \in B_{p, 1}^{\frac{N}{p}}, u_{0} \in B_{p_{1}, 1}^{\frac{N}{p_{1}}-1} \text { and } f \in L_{l o c}^{1}\left(\mathbb{R}^{+}, \in B_{p_{1}, 1}^{\frac{N}{p_{1}}-1}\right)
$$

with $\left(p, p_{1}\right) \in[1,+\infty[$ suitably chosen.

Recall that in $[25,24]$, D. Hoff stated the existence of global weak solutions with small initial data including discontinuous initial data (namely $q_{0}$ is small in $L^{2} \cap L^{\infty}$ and $u_{0}$ is small in $L^{4}$ if $N=2$ and small in $L^{8}$ if $N=3$ ). One of the major interest of the results of Hoff is to exhibit some smoothing effects on the incompressible part of the velocity $u$ and on the so-called effective viscous flux $F=(2 \mu+\lambda) \operatorname{div} u-P(\rho)+P(\bar{\rho})$ (see also the work by D. Serre in [40]). This also plays a crucial role in the proof of P-L Lions for the existence of global weak solution (see [34]). However if the results of Hoff are critical in the sense of the scaling for the density, it is not the case for the initial velocity. In [28, 29], 
D. Hoff extends the previous results by considering simply slightly subcritical initial data on the velocity whereas the initial density belongs always to $L^{\infty}$ (see also [30]). In [26], D. Hoff shows a very interesting theorem of weak-strong uniqueness when $P(\rho)=K \rho$ with $K>0$. To speak roughly, he shows that if $(\rho, u),\left(\rho_{1}, u_{1}\right)$ satisfy a $L^{\infty}$ control on the density combined with a Lipschitz control on the velocity then $(\rho, u)=\left(\rho_{1}, u_{1}\right)$. D. Hoff uses this result to show that the solutions of [28] are unique in two dimension. In [22], we prove a new criterion of blow-up for compressible Navier-Stokes equations of Prodi-Serrin type, where we show that the good unknown to control is the pressure term and not the velocity as for incompressible Navier-Stokes equations. More precisely the weak solutions of compressible Navier-Stokes equations turn out to be smooth as long as the density remains bounded in $L^{\infty}\left(L^{(N+1+\varepsilon) \gamma}\right)$ with $\varepsilon>0$ arbitrary small (a crucial part of the proof is based on the notion of effective velocity which possesses some additional properties of regularity compared with the classical velocity). Finally R. Danchin in [14] shows for the first time a result of existence of global strong solution close to a stable equilibrium in critical space for the scaling of the system. More precisely the initial data are chosen as follows $\left(q_{0}, u_{0}\right) \in\left(B_{2,1}^{\frac{N}{2}} \cap B_{2,1}^{\frac{N}{2}-1}\right) \times B_{2,1}^{\frac{N}{2}-1}$. The main difficulty is to get estimates on the linearized system given that the velocity and the density are coupled via the pressure. What is crucial in this work is the smoothing effect on the velocity and a $L^{1}$ decay on $\rho-\bar{\rho}$ (this plays a key role to control the pressure term). In this work, R. Danchin uses some clever energy inequalities on the system in Fourier variable. This explains in particular why the result is obtained in Besov space with a Lebesgue index $p=2$. In the same time of the redaction of this paper, Q. Chen et al in [11] and F. Charve and R. Danchin in [7] improve the previous result by working in more general Besov space.

The goal of this article is to make a connection between the articles of D. Hoff $[25,24$, 28, 29], D. Hoff and M. Santos [30] and those of F. Charve and R. Danchin and Q. Chen et al in [7] and [11]. In fact we extend the results [7] and [11] to the case where the Lebesgue index of Besov spaces are not the same for the density and the velocity. To do that, as in [21] we use the notion of effective velocity (also used by D. Hoff in [24], see the variable $u_{P}$ in his work which is not exactly our effective velocity but more precisely the velocity $v$ that we will define in the sequel) in high frequencies so as to kill the relation of coupling between the velocity and the pressure. This effective velocity enables us to get as in R. Danchin in [14] a $L^{1}$ decay on $q$ in the high frequency regime. In low frequencies, the first order terms predominate, so that (1.1) has to be treated by means of hyperbolic energy methods (roughly $q$ and the potential part of the velocity verify a wave equation). This implies that we can treat the low regime only in spaces constructed on $L^{2}$ as it is classical that hyperbolic systems are ill-posed in general $L^{p}$ spaces. So as in [11] and [7], the system has to be handled differently in low and high frequencies. In short, we will use the analysis of R. Danchin in [14] in low frequencies and the effective velocity introduced in [21] in high frequencies.

To simplify the notation, we assume from now on that $\bar{\rho}=1$. Hence as long as $\rho$ does not vanish, the equations for $(q=\rho-1, u)$ read:

$$
\left\{\begin{array}{l}
\partial_{t} q+u \cdot \nabla q=-(1+q) \operatorname{div} u \\
\partial_{t} u+u \cdot \nabla u-\frac{1}{1+q} \mathcal{A} u+\frac{\nabla P(1+q)}{1+q}=f
\end{array}\right.
$$


In the sequel we will note $\mathcal{A}=\mu \Delta+(\lambda+\mu) \nabla$ div.

Definition 1.2 Furthermore we will note $\widetilde{B}_{\left(p_{1}, r_{1}\right),\left(p_{2}, r_{2}\right)}^{s_{1}, s_{2}}$ the Besov space where the behavior is $B_{p_{1}, r_{1}}^{s_{1}}$ in low frequencies and $B_{p_{2}, r_{2}}^{s_{2}}$ in high frequencies. If $r_{1}=r_{2}$ we will simplify the notation, and we will write $\widetilde{B}_{p_{1}, p_{2}, 1}^{s_{1}, s_{2}}$ for $\widetilde{B}_{\left(p_{1}, 1\right),\left(p_{2}, 1\right)}^{s_{1}, s_{2}}$. For more details on the definition of these spaces we refer to the definition 2.6.

One can now state our main result.

Theorem 1.1 Let $P$ be a suitably smooth function of the density such that $P^{\prime}(1)>0$, and $1 \leq p_{1} \leq p<+\infty$ with $\frac{1}{p_{1}} \leq \frac{1}{N}+\frac{1}{p}, \frac{1}{p}+\frac{1}{p_{1}}>\frac{1}{N}, p<\max (4, N)$ and $\frac{1}{2} \leq \frac{1}{p}+\frac{1}{p_{1}}$. Assume that $u_{0} \in \widetilde{B}_{2, p_{1}, 1}^{\frac{N}{2}-1, \frac{N}{p_{1}}-1}, f \in L^{1}\left(\mathbb{R}^{+}, \widetilde{B}_{2, p_{1}, 1}^{\frac{N}{2}-1, \frac{N}{p_{1}}-1}\right)$ and $q_{0} \in \widetilde{B}_{2, p, 1}^{\frac{N}{2}-1, \frac{N}{p}}$. Then there exists a constant $\varepsilon_{0}$ such that if:

$$
\left\|q_{0}\right\|_{\widetilde{B}_{2, p, 1}^{\frac{N}{2}-1, \frac{N}{p}}}+\left\|u_{0}\right\|_{\widetilde{B}_{2, p_{1}, 1}^{\frac{N}{2}-1, \frac{N}{p_{1}}-1}}+\|f\|_{L^{1}\left(\mathbb{R}^{+}, \widetilde{B}_{2, p_{1}, 1}^{\frac{N}{2}-1, \frac{N}{p_{1}}-1}\right)} \leq \varepsilon_{0},
$$

then there exists a global solution $(q, u)$ for system (1.1) with $1+q$ bounded away from zero and,

$$
\begin{aligned}
q \in \widetilde{C}\left(\mathbb{R}^{+}, \widetilde{B}_{2, p, 1}^{\frac{N}{2}-1, \frac{N}{p}}\right) \cap L^{1}\left(\mathbb{R}^{+}, \widetilde{B}_{2, p, 1}^{\frac{N}{2}+1, \frac{N}{p}}\right) \text { and } \\
\\
\quad u \in \widetilde{C}\left(\mathbb{R}^{+} ; \widetilde{B}_{2, p_{1}, 1}^{\frac{N}{2}-1, \frac{N}{p_{1}}-1}+\widetilde{B}_{2, p, 1}^{\frac{N}{2}-1, \frac{N}{p}}\right) \cap L^{1}\left(\mathbb{R}+, \widetilde{B}_{2, p, 1}^{\frac{N}{2}+1, \frac{N}{p}+1}\right) .
\end{aligned}
$$

Moreover this solution is unique if $\frac{2}{N} \leq \frac{1}{p}+\frac{1}{p_{1}}$.

Remark 1 This theorem improves the result of Chen et al obtained in [11] as here one may take $p \neq p_{1}$. But as in [11] we have strong restrictions on $p$, that is $p<\max (4,2 N)$. This fact is due to the interactions between low and high frequencies in the paraproduct laws. However we obtain this result with a new method which seems more flexible ( see theorem 1.2) and more simple than [7] and [11].

Remark 2 It seems possible to improve the theorem 1.1 by choosing initial data $q_{0}$ in $B_{(2,1),(p, \infty)}^{\frac{N}{2}-1, \frac{N}{p}} \cap B_{(2,1),(\infty, 1)}^{\frac{N}{2}-1,0}$, however some supplementary conditions appear on $p_{1}$ in this case.

The key to theorem 1.1 is to introduce a new unknown $v_{1}$ to avoid the coupling between the density and the velocity, we analyze by a new way the pressure term. More precisely we write the gradient of the pressure as a Laplacian of some vector-field $v$ (it means that $\Delta v=\nabla P(\rho))$, and we introduce this term in the linear part of the momentum equation ( in other words, $v=\mathcal{G} P(\rho)$ where $\mathcal{G} P(\rho)$ stands for some pseudo-differential operator of order -1$)$. We then introduce the effective velocity $v_{1}=u-v$. By this way, we have canceled out the coupling between $v_{1}$ and the density. More precisely we have then from (1.3) the following system:

$$
\left\{\begin{aligned}
& \partial_{t} q+\left(v_{1}+\frac{1}{\nu} v\right) \cdot \nabla q+\frac{1}{\nu} P^{\prime}(1) q=-(1+q) \operatorname{div} v_{1} \\
&-\frac{1}{\nu}\left(P(\rho)-P(1)-P^{\prime}(1)\right)-\frac{1}{\nu} q(P(\rho)-P(1)), \\
& \partial_{t} v_{1}-\frac{1}{1+q} \mathcal{A} v_{1}=f-u \cdot \nabla u+\frac{1}{\nu} \nabla(\Delta)^{-1}\left(P^{\prime}(\rho) \operatorname{div}(\rho u)\right), \\
& q_{/ t=0}=a_{0},\left(v_{1}\right)_{/ t=0}=\left(v_{1}\right)_{0},
\end{aligned}\right.
$$


where $v_{1}=u-\frac{1}{\nu} v$ is called the effective velocity. Here we can check that $v_{1}$ verifies a parabolic equations with the low order terms in remains $\nabla(\Delta)^{-1}\left(P^{\prime}(\rho) \operatorname{div}(\rho u)\right.$ while $q$ satisfies a damped transport equation. We next verify easily that we have a Lipschitz control on the gradient of $u$ (it is crucial to estimate the density by the mass equation). For more details on the strategy of the proof we refer to section 4.1.

In the previous theorem 1.1, we have as in [11] very strong restrictions on $p(p<$ $\max (4,2 N))$ because of the behavior in low frequencies. At the difference with the results of strong solutions in finite time (see [21]), we cannot choose $p$ arbitrarly large. To overcome this difficulty, we need to add some additional conditions on $\left(q_{0}, u_{0}\right)$ in low frequencies as in [7] to avoid these restrictions in the use of the paraproduct laws. We obtain then the following theorem:

Theorem 1.2 Let $P$ be a suitably smooth function of the density with $P^{\prime}(1)>0$ and $1 \leq$ $p_{1} \leq p<+\infty$ such that $\frac{1}{p_{1}} \leq \frac{1}{N}+\frac{1}{p}$ and $\frac{1}{p}+\frac{1}{p_{1}}>\frac{1}{N}$. Assume that $u_{0} \in \widetilde{B}_{2, p_{1}, 1}^{\frac{N}{2}-1, \frac{N}{p_{1}}-1} \cap B_{2, r}^{0}$, $f \in \widetilde{L}^{1}\left(\mathbb{R}^{+}, \widetilde{B}_{2, p_{1}, 1}^{\frac{N}{2}-1, \frac{N}{p_{1}}-1} \cap B_{2, r}^{0}\right)$ and $q_{0} \in \widetilde{B}_{2, p, 1}^{\frac{N}{2}-1, \frac{N}{p}} \cap \widetilde{B}_{2, r}^{0,1}$ with $r=+\infty$ if $N \geq 3$ and $r=1$ if $N=2$. Then there exists a constant $\varepsilon_{0}$ such that if:

$$
\left\|q_{0}\right\|_{\widetilde{B}_{2, p, 1}^{\frac{N}{2}-1, \frac{N}{p}} \cap \widetilde{B}_{2, r}^{0,1}}+\left\|u_{0}\right\|_{\widetilde{B}_{2, p_{1}, 1}^{\frac{N}{2}-1, \frac{N}{p_{1}}-1} \cap B_{2, r}^{0}}+\|f\|_{\widetilde{L}^{1}\left(\mathbb{R}^{+}, \widetilde{B}_{2, p_{1}, 1}^{\frac{N}{2}-1, \frac{N}{p_{1}}-1} \cap B_{2, r}^{0}\right)} \leq \varepsilon_{0},
$$

then there exists a global solution $(q, u)$ for system (1.1) with $1+q$ bounded away from zero and,

$$
\begin{aligned}
q \in \widetilde{C}\left(\mathbb{R}^{+}, \widetilde{B}_{2, p, 1}^{\frac{N}{2}-1, \frac{N}{p}} \cap \widetilde{B}_{2, r}^{0,1}\right) \cap \widetilde{L}^{1}\left(\mathbb{R}^{+}, \widetilde{B}_{2, p, 1}^{\frac{N}{2}+1, \frac{N}{p}} \cap \widetilde{B}_{2, r}^{2,1}\right) \quad \text { and } \\
\\
\quad u \in \widetilde{C}\left(\mathbb{R}^{+} ;\left(\widetilde{B}_{2, p_{1}, 1}^{\frac{N}{2}-1, \frac{N}{p_{1}}-1}+\widetilde{B}_{2, p, 1}^{\frac{N}{2}-1, \frac{N}{p}}\right) \cap B_{2, r}^{0}\right) \cap \widetilde{L}^{1}\left(\mathbb{R}^{+}, \widetilde{B}_{2, p, 1}^{\frac{N}{2}+1, \frac{N}{p}+1} \cap B_{2, r}^{2}\right) .
\end{aligned}
$$

Moreover this solution is unique if $\frac{2}{N} \leq \frac{1}{p}+\frac{1}{p_{1}}$.

Remark 3 Here we improve the results of [7] and [11] by the fact that we have no restriction on the size of $p$ of type $p<2 N$. In particular, we can observe that when $p$ goes to infinity, we are close to get a weak solution with initial data $\left(q_{0}, u_{0}\right)$ in $\widetilde{B}_{2, \infty, 1}^{\frac{N}{2}-1,0} \times$ $\widetilde{B}_{2, N, 1}^{\frac{N}{2}-1,1}$. It means that this theorem bridges the gap of the result of D. Hoff (see [25, 26, 28, 29] where the initial density is in $L^{\infty}$ but where the initial velocity is more regular and the results of $R$. Danchin in [17]. Moreover it holds for general pressure laws with $P^{\prime}(1)>0$ while in the works of $D$. Hoff the pressure has to verify $P(\rho)=K \rho$ with $K>0$.

Remark 4 If $r=+\infty$, then we have to replace above the strong continuity in $B_{2, r}^{s}$ by the weak continuity.

Remark 5 To some extent the case $p>N$ is not so important because we obtain only the existence of global weak solution as in the works of D. Hoff in [25, 26]. However as in the work of F. Charve and R. Danchin in [7] it is of interest, indeed by adding some additional condition on the data such that $u_{0} \in B_{N, 1}^{0}$ (without assumption of smallness on $\left\|u_{0}\right\|_{B_{N, 1}^{0}}$ ) it is easy to prove some results of persistency on $u$ as for incompressible Navier-Stokes 
equations, more precisely that $u$ belongs to $\widetilde{L}^{\infty}\left(\widetilde{B}_{2, N, 1}^{\frac{N}{2}-1,0}+\widetilde{B}_{2, p, 1}^{\frac{N}{2}-1, \frac{N}{p}}\right) \cap \widetilde{L}^{1}\left(\widetilde{B}_{2, p, 1}^{\frac{N}{2}+1, \frac{N}{p}+1}\right)$. In this case, we will get the existence of global strong solutions but with only a condition of smallness for $\left\|q_{0}\right\|_{\widetilde{B}_{2, p, 1}^{\frac{N}{2}-1, \frac{N}{p}} \cap B_{2, r}^{0,1}},\left\|u_{0}\right\|_{\widetilde{B}_{2, p_{1}, 1}^{\frac{N}{2}-1, \frac{N}{p_{1}}-1} \cap B_{2, r}^{0}}$ and $\|f\|_{\widetilde{L}^{1}\left(\mathbb{R}^{+}, \widetilde{B}_{2, p_{1}, 1}^{\frac{N}{2}-1, \frac{N}{p_{1}}-1} \cap B_{2, r}^{0}\right)}$ but not on $\left\|u_{0}\right\|_{B_{N, 1}^{0}}$.

In particular it improves widely the results of [11] and [7] inasmuch as we can choose $p$ as large that we want. Therefore one can almost handle the case $q_{0} \in B_{\infty, 1}^{0}$. Hence we are close to the results of D. Hoff in [25, 28, 29] in terms of initial density (a bit more regular) except that we really work in critical spaces for the scaling of the equations in particular concerning the initial velocity. Furthermore we are able to build unique solutions when $p$ is arbitrarily large by adding a condition on $u_{0} \in B_{N, 1}^{0}$ (without assumption of smallness on $\left.\left\|u_{0}\right\|_{B_{N, 1}^{0}}\right)$. The only point is that we need to ask a condition of regularity on $q_{0} \in \widetilde{B}_{2, r}^{0,1}$ (we will see how to deal with this problem in some case, see remark 7).

In particular as in [7], we can take $u_{0}(x)=\phi(x) \sin \left(\varepsilon^{-1} x \cdot \omega\right) n$ where $\omega$ and $n$ stand for any unit vector in $\mathbb{R}^{N}$ and $\phi$ for any smooth compactly supported function then we have if $p_{1}>N$, and for $s<0$ :

$$
\left\|u_{0}\right\|_{B_{p_{1}, 1}^{\frac{N}{p_{1}}-1}} \leq C \varepsilon^{1-\frac{N}{p_{1}}} \quad \text { and }\left\|u_{0}\right\|_{B_{2, r}^{s}} \leq C \varepsilon^{-s},
$$

so that the smallness condition is satisfied by $u_{0}$ if $\varepsilon$ small enough. However we remark that $u_{0}$ is arbitrarily big in $L^{3}$. On the other hand, $u_{0}$ belongs to Schwartz class $\mathcal{S}$ hence also in $B_{N, 1}^{0}$ so that uniqueness holds true by persistency results.

In our case, we can not directly apply the previous theorem because we work with $u_{0} \in B_{2, r}^{0}$ (it means that in this case with the above choice on $\left\|u_{0}\right\|_{B_{2, r}^{0}}$ is not small). We have then to modify the assumption on $u_{0}$ to obtain the same type of result than [7]. In fact, it is enough to assume that $u_{0} \in B_{2, r}^{-s_{1}}$ and $q \in B_{2, r}^{-s_{1}, 1-s_{1}}$ with $s_{1}>0$ arbitrary small, in this case we have some new limitation on the choice of $p$ in function of $s_{1}$ (because the law of paraproduct). However we can easily check that $p$ goes to infinity when $s_{1}$ goes to 0. So we can get the result of [7] by choosing again arbitrarily large $p$.

Remark 6 We can observe that $u_{0} \in B_{2, r}^{0}$ corresponds exactly to the energy space when $r=2$, in this sense this additional assumption on the velocity seems very natural. However the condition $q_{0} \in \widetilde{B}_{2, r}^{0,1}$ seems a little bit too strong in the general case for general viscosity coefficients, but we will explain in the theorem 1.3 why in some specific case of viscosity coefficients this condition is suitable.

We finally treat the case of variable viscosity coefficients. More particularly we are interested in considering the specific case of the so-called BD viscosity coefficients (see $[5,6]$ ). Indeed with this choice, we naturally obtain some informations on $q$ in $B_{2,2}^{1}=H^{1}$. In this context, the hypothesis of theorem 1.2 on $q_{0} \in \widetilde{B}_{2, r}^{0,1}$ becomes natural (for more explanations see remark 7 ).

In the following theorem, we then extend the theorem 1.2 to the case of general viscosity coefficients.

Theorem 1.3 Let $P$ be a suitably smooth function of the density with $P^{\prime}(1)>0, \mu$ and $\lambda$ are general regular functions such that $\mu>0$ and $2 \mu+\lambda>0$. Furthermore we 
suppose that $1 \leq p_{1} \leq p<+\infty$ such that $\frac{1}{p_{1}} \leq \frac{1}{N}+\frac{1}{p}$ and $\frac{1}{p}+\frac{1}{p_{1}}>\frac{1}{N}$. Assume that $u_{0} \in \widetilde{B}_{2, p_{1}, 1}^{\frac{N}{2}-1, \frac{N}{p_{1}}-1} \cap B_{2, r}^{0}, f \in \widetilde{L}^{1}\left(\mathbb{R}^{+}, \widetilde{B}_{2, p_{1}, 1}^{\frac{N}{2}-1, \frac{N}{p_{1}}-1} \cap B_{2, r}^{0}\right)$ and $q_{0} \in \widetilde{B}_{2, p, 1}^{\frac{N}{2}-1, \frac{N}{p}} \cap \widetilde{B}_{2, r}^{0,1}$ with $r=+\infty$ if $N \geq 3$ and $r=1$ if $N=2$. Then there exists a constant $\varepsilon_{0}$ such that if:

$$
\left\|q_{0}\right\|_{\widetilde{B}_{2, p, 1}^{\frac{N}{2}-1, \frac{N}{p}} \cap \widetilde{B}_{2, r}^{0,1}}+\left\|u_{0}\right\|_{\widetilde{B}_{2, p_{1}, 1}^{\frac{N}{2}-1, \frac{N}{p_{1}}-1} \cap B_{2, r}^{0}}+\|f\|_{\widetilde{L}^{1}\left(\mathbb{R}^{+}, \widetilde{B}_{2, p_{1}, 1}^{\frac{N}{2}-1, \frac{N}{p_{1}}-1} \cap B_{2, r}^{0}\right)} \leq \varepsilon_{0},
$$

there exists a global solution $(q, u)$ for system (1.1) with $1+q$ bounded away from zero and,

$$
\begin{aligned}
q \in \widetilde{C}\left(\mathbb{R}^{+}, \widetilde{B}_{2, p, 1}^{\frac{N}{2}-1, \frac{N}{p}} \cap \widetilde{B}_{2, r}^{0,1}\right) \cap \widetilde{L}^{1}\left(\mathbb{R}^{+}, \widetilde{B}_{2, p, 1}^{\frac{N}{2}+1, \frac{N}{p}} \cap \widetilde{B}_{2, r}^{2,1}\right) \quad \text { and } \\
\quad u \in \widetilde{C}\left(\mathbb{R}^{+} ;\left(\widetilde{B}_{2, p_{1}, 1}^{\frac{N}{2}-1, \frac{N}{p_{1}}-1}+\widetilde{B}_{2, p, 1}^{\frac{N}{2}-1, \frac{N}{p}}\right) \cap B_{2, r}^{0}\right) \cap \widetilde{L}^{1}\left(\mathbb{R}^{+}, \widetilde{B}_{2, p, 1}^{\frac{N}{2}+1, \frac{N}{p}+1} \cap B_{2, r}^{2}\right) .
\end{aligned}
$$

Moreover this solution is unique if $\frac{2}{N} \leq \frac{1}{p}+\frac{1}{p_{1}}$.

Remark 7 This result is very interesting in the case of the BD viscosity coefficients. In this case our result is very close of the energy initial data with the optimal condition for the scaling $\left(q_{0}, u_{0}\right) \in B_{\infty, 1}^{0} \times B_{N, 1}^{0}$. In particular it applies to the shallow-water system. Indeed in [5, 6] D. Bresch and B. Desjardins have discovered a new entropy inequality whenever the density-dependent viscosity coefficients satisfy the algebraic relation:

$$
\lambda(\rho)=\rho \mu^{\prime}(\rho)-\mu(\rho) .
$$

In this case they show that we can control $\sqrt{\rho} \nabla \varphi(\rho)$ in $L^{\infty}\left(L^{2}\right)$ where $\varphi^{\prime}(\rho)=\frac{\mu^{\prime}(\rho)}{\rho}$. Roughly it means that we control the density $\rho$ in $L^{\infty}\left(H^{1}\right)$. It is exactly the additional condition that we ask in the theorems 1.2 and 1.3.

We would like to point out that D. Bresch and B. Desjardins use a type of effective velocity that they note $u+\nabla \varphi(\rho)$ (see [5] for more details) different from the one that we introduce in this paper.

Remark 8 Our method is more flexible than the proofs of D. Hoff in [26, 25, 28, 29] as these works are based crucially on the notion of effective pressure and on a gain of integrability on the velocity which works only in the case of constant viscosity coefficients. Indeed the used technics are essentially based on energy inequalities that is why the fact to work with constant viscosity coefficients is crucial.

Our paper is structured as follows. In section 2, we give a few notation and briefly introduce the basic Fourier analysis techniques needed to prove our result. In section 3, we prove estimates on the transport equation. In section 4, we prove the theorem 1.1. In section 5 we prove the theorems 1.2 and 1.3. Some technical continuity results for the paraproduct in hybrid Besov spaces have been postponed in appendix.

\section{Littlewood-Paley theory and Besov spaces}

Throughout the paper, $C$ stands for a constant whose exact meaning depends on the context. The notation $A \lesssim B$ means that $A \leq C B$. For all Banach space $X$, we 
denote by $C([0, T], X)$ the set of continuous functions on $[0, T]$ with values in $X$. For $p \in[1,+\infty]$, the notation $L^{p}(0, T, X)$ or $L_{T}^{p}(X)$ stands for the set of measurable functions on $(0, T)$ with values in $X$ such that $t \rightarrow\|f(t)\|_{X}$ belongs to $L^{p}(0, T)$. Littlewood-Paley decomposition corresponds to a dyadic decomposition of the space in Fourier variables. We can use for instance any $\varphi \in C^{\infty}\left(\mathbb{R}^{N}\right)$, supported in $\mathcal{C}=\left\{\xi \in \mathbb{R}^{N} / \frac{3}{4} \leq|\xi| \leq \frac{8}{3}\right\}$ such that:

$$
\sum_{l \in \mathbb{Z}} \varphi\left(2^{-l} \xi\right)=1 \text { if } \xi \neq 0 .
$$

Denoting $h=\mathcal{F}^{-1} \varphi$, we then define the dyadic blocks by:

$$
\Delta_{l} u=\varphi\left(2^{-l} D\right) u=2^{l N} \int_{\mathbb{R}^{N}} h\left(2^{l} y\right) u(x-y) d y \text { and } S_{l} u=\sum_{k \leq l-1} \Delta_{k} u .
$$

Formally, one can write that:

$$
u=\sum_{k \in \mathbb{Z}} \Delta_{k} u
$$

This decomposition is called homogeneous Littlewood-Paley decomposition. Let us observe that the above formal equality does not hold in $\mathcal{S}^{\prime}\left(\mathbb{R}^{N}\right)$ for two reasons:

1. The right hand-side does not necessarily converge in $\mathcal{S}^{\prime}\left(\mathbb{R}^{N}\right)$.

2. Even if it does, the equality is not always true in $\mathcal{S}^{\prime}\left(\mathbb{R}^{N}\right)$ (consider the case of the polynomials).

\subsection{Homogeneous Besov spaces and first properties}

Definition 2.3 For $s \in \mathbb{R}, p \in[1,+\infty], q \in[1,+\infty]$, and $u \in \mathcal{S}^{\prime}\left(\mathbb{R}^{N}\right)$ we set:

$$
\|u\|_{B_{p, q}^{s}}=\left(\sum_{l \in \mathbb{Z}}\left(2^{l s}\left\|\Delta_{l} u\right\|_{L^{p}}\right)^{q}\right)^{\frac{1}{q}} .
$$

The Besov space $B_{p, q}^{s}$ is the set of temperate distribution $u$ such that $\|u\|_{B_{p, q}^{s}}<+\infty$.

Remark 9 The above definition is a natural generalization of the nonhomogeneous Sobolev and Hölder spaces: one can show that $B_{\infty, \infty}^{s}$ is the nonhomogeneous Hölder space $C^{s}$ and that $B_{2,2}^{s}$ is the nonhomogeneous space $H^{s}$.

Proposition 2.1 The following properties holds:

1. there exists a constant universal $C$ such that: $C^{-1}\|u\|_{B_{p, r}^{s}} \leq\|\nabla u\|_{B_{p, r}^{s-1}} \leq C\|u\|_{B_{p, r}^{s}}$.

2. If $p_{1}<p_{2}$ and $r_{1} \leq r_{2}$ then $B_{p_{1}, r_{1}}^{s} \hookrightarrow B_{p_{2}, r_{2}}^{s-N\left(1 / p_{1}-1 / p_{2}\right)}$.

3. $B_{p, r_{1}}^{s^{\prime}} \hookrightarrow B_{p, r}^{s}$ if $s^{\prime}>s$ or if $s=s^{\prime}$ and $r_{1} \leq r$. 
Let now recall a few product laws in Besov spaces coming directly from the paradifferential calculus of J-M. Bony (see [4]) and rewrite on a generalized form in [1] by H. Abidi and M. Paicu (in this article the results are written in the case of homogeneous sapces but it can easily generalize for the nonhomogeneous Besov spaces).

Proposition 2.2 We have the following laws of product:

- For all $s \in \mathbb{R},(p, r) \in[1,+\infty]^{2}$ we have:

$$
\|u v\|_{B_{p, r}^{s}} \leq C\left(\|u\|_{L^{\infty}}\|v\|_{B_{p, r}^{s}}+\|v\|_{L^{\infty}}\|u\|_{B_{p, r}^{s}}\right) .
$$

- Let $\left(p, p_{1}, p_{2}, r, \lambda_{1}, \lambda_{2}\right) \in[1,+\infty]^{2}$ such that: $\frac{1}{p} \leq \frac{1}{p_{1}}+\frac{1}{p_{2}}, p_{1} \leq \lambda_{2}, p_{2} \leq \lambda_{1}, \frac{1}{p} \leq$ $\frac{1}{p_{1}}+\frac{1}{\lambda_{1}}$ and $\frac{1}{p} \leq \frac{1}{p_{2}}+\frac{1}{\lambda_{2}}$. We have then the following inequalities:

if $s_{1}+s_{2}+N \inf \left(0,1-\frac{1}{p_{1}}-\frac{1}{p_{2}}\right)>0, s_{1}+\frac{N}{\lambda_{2}}<\frac{N}{p_{1}}$ and $s_{2}+\frac{N}{\lambda_{1}}<\frac{N}{p_{2}}$ then:

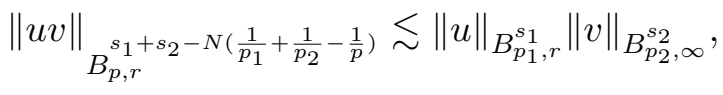

when $s_{1}+\frac{N}{\lambda_{2}}=\frac{N}{p_{1}}$ (resp $\left.s_{2}+\frac{N}{\lambda_{1}}=\frac{N}{p_{2}}\right)$ we replace $\|u\|_{B_{p_{1}, r}^{s_{1}}}\|v\|_{B_{p_{2}, \infty}^{s_{2}}}\left(\right.$ resp $\left.\|v\|_{B_{p_{2}, \infty}^{s_{2}}}\right)$

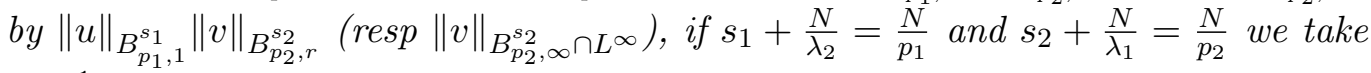
$r=1$.

If $s_{1}+s_{2}=0, s_{1} \in\left(\frac{N}{\lambda_{1}}-\frac{N}{p_{2}}, \frac{N}{p_{1}}-\frac{N}{\lambda_{2}}\right]$ and $\frac{1}{p_{1}}+\frac{1}{p_{2}} \leq 1$ then:

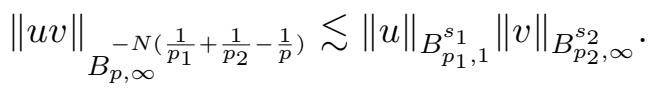

$$
\begin{aligned}
& \text { If }|s|<\frac{N}{p} \text { for } p \geq 2 \text { and }-\frac{N}{p^{\prime}}<s<\frac{N}{p} \text { else, we have: } \\
& \qquad\|u v\|_{B_{p, r}^{s}} \leq C\|u\|_{B_{p, r}^{s}}\|v\|_{B_{p, \infty}^{\frac{N}{p}} \cap L^{\infty}} .
\end{aligned}
$$

Remark 10 In the sequel $p$ will be either $p_{1}$ or $p_{2}$ and in this case $\frac{1}{\lambda}=\frac{1}{p_{1}}-\frac{1}{p_{2}}$ if $p_{1} \leq p_{2}$, resp $\frac{1}{\lambda}=\frac{1}{p_{2}}-\frac{1}{p_{1}}$ if $p_{2} \leq p_{1}$.

Corollary 1 Let $r \in[1,+\infty], 1 \leq p \leq p_{1} \leq+\infty$ and $s$ such that:

- $s \in\left(-\frac{N}{p_{1}}, \frac{N}{p_{1}}\right)$ if $\frac{1}{p}+\frac{1}{p_{1}} \leq 1$,

- $s \in\left(-\frac{N}{p_{1}}+N\left(\frac{1}{p}+\frac{1}{p_{1}}-1\right), \frac{N}{p_{1}}\right)$ if $\frac{1}{p}+\frac{1}{p_{1}}>1$,

then we have if $u \in B_{p, r}^{s}$ and $v \in B_{p_{1}, \infty}^{\frac{N}{p_{1}}} \cap L^{\infty}$ :

$$
\|u v\|_{B_{p, r}^{s}} \leq C\|u\|_{B_{p, r}^{s}}\|v\|_{B_{p_{1}, \infty}^{\frac{N}{p_{1}}} \cap L^{\infty}} .
$$

The study of non stationary PDE's requires space of type $L^{\rho}(0, T, X)$ for appropriate Banach spaces $X$. In our case, we expect $X$ to be a Besov space, so that it is natural to localize the equation through Littlewood-Payley decomposition. But, in doing so, we obtain bounds in spaces which are not type $L^{\rho}(0, T, X)$ (except if $r=p$ ). We are now going to define the spaces of Chemin-Lerner in which we will work, which are a refinement of the spaces $L_{T}^{\rho}\left(B_{p, r}^{s}\right)$. 
Definition 2.4 Let $\rho \in[1,+\infty], T \in[1,+\infty]$ and $s_{1} \in \mathbb{R}$. We set:

$$
\|u\|_{\widetilde{L}_{T}^{\rho}\left(B_{p, r}^{s_{1}}\right)}=\left(\sum_{l \in \mathbb{Z}} 2^{l r s_{1}}\left\|\Delta_{l} u(t)\right\|_{L^{\rho}\left(L^{p}\right)}^{r}\right)^{\frac{1}{r}} .
$$

We then define the space $\widetilde{L}_{T}^{\rho}\left(B_{p, r}^{s_{1}}\right)$ as the set of temperate distribution u over $(0, T) \times \mathbb{R}^{N}$ such that $\|u\|_{\widetilde{L}_{T}^{\rho}\left(B_{p, r}^{\left.s_{1}\right)}\right.}<+\infty$.

We set $\widetilde{C}_{T}\left(\widetilde{B}_{p, r}^{s_{1}}\right)=\widetilde{L}_{T}^{\infty}\left(\widetilde{B}_{p, r}^{s_{1}}\right) \cap \mathcal{C}\left([0, T], B_{p, r}^{s_{1}}\right)$. Let us emphasize that, according to Minkowski inequality, we have:

$$
\|u\|_{\widetilde{L}_{T}^{\rho}\left(B_{p, r}^{s_{1}}\right)} \leq\|u\|_{L_{T}^{\rho}\left(B_{p, r}^{s_{1}}\right)} \text { if } r \geq \rho, \quad\|u\|_{\widetilde{L}_{T}^{\rho}\left(B_{p, r}^{s_{1}}\right)} \geq\|u\|_{L_{T}^{\rho}\left(B_{p, r}^{s_{1}}\right)} \text { if } r \leq \rho .
$$

Remark 11 It is easy to generalize proposition 2.2, to $\widetilde{L}_{T}^{\rho}\left(B_{p, r}^{s_{1}}\right)$ spaces. The indices $s_{1}$, $p, r$ behave just as in the stationary case whereas the time exponent $\rho$ behaves according to Hölder inequality.

In the sequel we will need of composition lemma in $\widetilde{L}_{T}^{\rho}\left(B_{p, r}^{s}\right)$ spaces.

Lemma 1 Let $s>0,(p, r) \in[1,+\infty]$ and $u \in \widetilde{L}_{T}^{\rho}\left(B_{p, r}^{s}\right) \cap L_{T}^{\infty}\left(L^{\infty}\right)$.

1. Let $F \in W_{l o c}^{[s]+2, \infty}\left(\mathbb{R}^{N}\right)$ such that $F(0)=0$. Then $F(u) \in \widetilde{L}_{T}^{\rho}\left(B_{p, r}^{s}\right)$. More precisely there exists a function $C$ depending only on $s, p, r, N$ and $F$ such that:

$$
\|F(u)\|_{\widetilde{L}_{T}^{\rho}\left(B_{p, r}^{s}\right)} \leq C\left(\|u\|_{L_{T}^{\infty}\left(L^{\infty}\right)}\right)\|u\|_{\widetilde{L}_{T}^{\rho}\left(B_{p, r}^{s}\right)} .
$$

2. Let $F \in W_{l o c}^{[s]+3, \infty}\left(\mathbb{R}^{N}\right)$ such that $F(0)=0$. Then $F(u)-F^{\prime}(0) u \in \widetilde{L}_{T}^{\rho}\left(B_{p, r}^{s}\right)$. More precisely there exists a function $C$ depending only on $s, p, r, N$ and $F$ such that:

$$
\left\|F(u)-F^{\prime}(0) u\right\|_{\widetilde{L}_{T}^{\rho}\left(B_{p, r}^{s}\right)} \leq C\left(\|u\|_{L_{T}^{\infty}\left(L^{\infty}\right)}\right)\|u\|_{\widetilde{L}_{T}^{\rho}\left(B_{p, r}^{s}\right)}^{2} .
$$

Now we give some result on the behavior of the Besov spaces via some pseudodifferential operator (see [12]).

Definition 2.5 Let $m \in \mathbb{R}$. A smooth function function $f: \mathbb{R}^{N} \rightarrow \mathbb{R}$ is said to be a $\mathcal{S}^{m}$ multiplier if for all muti-index $\alpha$, there exists a constant $C_{\alpha}$ such that:

$$
\forall \xi \in \mathbb{R}^{N},\left|\partial^{\alpha} f(\xi)\right| \leq C_{\alpha}(1+|\xi|)^{m-|\alpha|} .
$$

Proposition 2.3 Let $m \in \mathbb{R}$ and $f$ be a $\mathcal{S}^{m}$ multiplier. Then for all $s \in \mathbb{R}$ and $1 \leq$ $p, r \leq+\infty$ the operator $f(D)$ is continuous from $B_{p, r}^{s}$ to $B_{p, r}^{s-m}$.

Let us now give some estimates for the heat equation: 
Proposition 2.4 Let $s \in \mathbb{R},(p, r) \in[1,+\infty]^{2}$ and $1 \leq \rho_{2} \leq \rho_{1} \leq+\infty$. Assume that $u_{0} \in B_{p, r}^{s}$ and $f \in \widetilde{L}_{T}^{\rho_{2}}\left(B_{p, r}^{s-2+2 / \rho_{2}}\right)$. Let $u$ be a solution of:

$$
\left\{\begin{array}{l}
\partial_{t} u-\mu \Delta u=f \\
u_{t=0}=u_{0}
\end{array}\right.
$$

Then there exists $C>0$ depending only on $N, \mu, \rho_{1}$ and $\rho_{2}$ such that:

$$
\|u\|_{\widetilde{L}_{T}^{\rho_{1}}\left(\widetilde{B}_{p, r}^{s+2 / \rho_{1}}\right)} \leq C\left(\left\|u_{0}\right\|_{B_{p, r}^{s}}+\mu^{\frac{1}{\rho_{2}}-1}\|f\|_{\widetilde{L}_{T}^{\rho_{2}\left(B_{p, r}^{s+2}\right.}}{ }^{\left.s-2 / \rho_{2}\right)}\right) .
$$

If in addition $r$ is finite then $u$ belongs to $C\left([0, T], B_{p, r}^{s}\right)$.

We now want to conclude by a useful lemma of commutator.

Lemma 2 Let $1 \leq p_{1} \leq p \leq+\infty$ and $\sigma \in\left(-\min \left(\frac{N}{p}, \frac{N}{p_{1}^{\prime}}\right), \frac{N}{p}+1\right]$. There exists a sequence $c_{q} \in l^{1}(\mathbb{Z})$ such that $\left\|c_{q}\right\|_{l^{1}}=1$ and a constant $C$ depending only on $N$ and $\sigma$ such that:

$$
\forall q \in \mathbb{Z}, \quad\left\|\left[v \cdot \nabla, \Delta_{q}\right] a\right\|_{L^{p_{1}}} \leq C c_{q} 2^{-q \sigma}\|\nabla v\|_{B_{p, 1}^{\frac{N}{p}}}\|a\|_{B_{p_{1}, 1}^{\sigma}} .
$$

In the limit case $\sigma=-\min \left(\frac{N}{p}, \frac{N}{p_{1}^{\prime}}\right)$, we have:

$$
\forall q \in \mathbb{Z}, \quad\left\|\left[v \cdot \nabla, \Delta_{q}\right] a\right\|_{L^{p_{1}}} \leq C c_{q} 2^{q \frac{N}{p}}\|\nabla v\|_{B_{p, 1}^{\frac{N}{p}}}\|a\|_{B_{p, \infty}^{-\frac{N}{p_{1}}}} \cdot
$$

Finally, for all $\sigma>0$ and $\frac{1}{p_{2}}=\frac{1}{p_{1}}-\frac{1}{p}$, there exists a constant $C$ depending only on $N$ and on $\sigma$ and a sequence $c_{q} \in l^{1}(\mathbb{Z})$ with norm 1 such that:

$\forall q \in \mathbb{Z}, \quad\left\|\left[v \cdot \nabla, \Delta_{q}\right] v\right\|_{L^{p}} \leq C c_{q} 2^{-q \sigma}\left(\|\nabla v\|_{L^{\infty}}\|v\|_{B_{p_{1}, 1}^{\sigma}}+\|\nabla v\|_{L^{p_{2}}}\|\nabla v\|_{B_{p, 1}^{\sigma-1}}\right)$.

Proof: These results are proved in [3] chapter 2.

\subsection{Hybrid Besov spaces}

The homogeneous Besov spaces fail to have nice inclusion properties: owing to the low frequencies, the embedding $B_{p, 1}^{s} \hookrightarrow B_{p, 1}^{t}$ does not hold for $s>t$. Still, the functions of $B_{p, 1}^{s}$ are locally more regular than those of $B_{p, 1}^{t}$ : for any $\phi \in C_{0}^{\infty}$ and $u \in B_{p, 1}^{s}$, the function $\phi u \in B_{p, 1}^{t}$. This motivates the definition of Hybrid Besov spaces introduced by R. Danchin in [14] where the growth conditions satisfied by the dyadic blocks and the coefficient of integrability are not the same for low and high frequencies. Hybrid Besov spaces have been used in [15] to prove global well-posedness for compressible gases in critical spaces. We generalize here a little bit the definition by allowing for different Lebesgue norms in low and high frequencies.

Definition 2.6 Let $l_{0} \in \mathbb{N}, s, t, \in \mathbb{R},\left(r, r_{1}\right) \in[1,+\infty]^{2}$ and $(p, q) \in[1,+\infty]$. We set:

$$
\|u\|_{\widetilde{B}_{p, q, 1}^{s, t}}=\sum_{l \leq l_{0}} 2^{l s}\left\|\Delta_{l} u\right\|_{L^{p}}+\sum_{l>l_{0}} 2^{l t}\left\|\Delta_{l} u\right\|_{L^{q}},
$$

and:

$$
\|u\|_{\left.\widetilde{B}_{(p, r),\left(q, r_{1}\right.}^{s, t}\right)}=\left(\sum_{l \leq l_{0}}\left(2^{l s}\left\|\Delta_{l} u\right\|_{L^{p}}\right)^{r}\right)^{\frac{1}{r}}+\left(\sum_{l>l_{0}}\left(2^{l t}\left\|\Delta_{l} u\right\|_{L^{q}}\right)^{r_{1}}\right)^{\frac{1}{r_{1}}} .
$$


Remark 12 It will be important in the sequel to chose $l_{0}$ big enough.

Notation 1 We will often use the following notation:

$$
u_{B F}=\sum_{l \leq l_{0}} \Delta_{l} u \quad \text { and } \quad u_{H F}=\sum_{l>l_{0}} \Delta_{l} u
$$

Remark 13 We have the following properties:

- We have $\widetilde{B}_{p, p, 1}^{s, s}=B_{p, 1}^{s}$.

- If $s_{1} \geq s_{3}$ and $s_{2} \geq s_{4}$ then $\widetilde{B}_{p, q, 1}^{s_{3}, s_{2}} \hookrightarrow \widetilde{B}_{p, q, 1}^{s_{1}, s_{4}}$.

We shall also make use of hybrid Besov-spaces. For them, one can prove results analoguous to proposition 2.2, we refer to proposition 6.8 in the appendix.

\section{The mass conservation equation}

We begin this section by recalling some estimates in Besov spaces for transport and heat equations. For more details, the reader is referred to [3].

Proposition 3.5 Let $1 \leq p_{1} \leq p \leq+\infty, r \in[1,+\infty]$ and $s \in \mathbb{R}$ be such that:

$$
-N \min \left(\frac{1}{p_{1}}, \frac{1}{p^{\prime}}\right)<s<1+\frac{N}{p_{1}} .
$$

Suppose that $q_{0} \in B_{p, r}^{s}, F \in L^{1}\left(0, T, B_{p, r}^{s}\right)$ and that $q \in L_{T}^{\infty}\left(B_{p, r}^{s}\right) \cap C\left([0, T] ; \mathcal{S}^{\prime}\right)$ solves the following transport equation:

$$
\left\{\begin{array}{l}
\partial_{t} q+u \cdot \nabla q=F \\
q_{t=0}=q_{0}
\end{array}\right.
$$

There exists a constant $C$ depending only on $N, p, p_{1}, r$ and $s$ such that, we have for a.e $t \in[0, T]$ :

$$
\|q\|_{\widetilde{L}_{t}^{\infty}\left(B_{p, r}^{s}\right)} \leq e^{C U(t)}\left(\left\|q_{0}\right\|_{B_{p, r}^{s}}+\int_{0}^{t} e^{-C U(\tau)}\|F(\tau)\|_{B_{p, r}^{s}} d \tau\right),
$$

with: $U(t)=\int_{0}^{t}\|\nabla u(\tau)\|_{B_{p_{1}, \infty}^{\frac{N}{p_{1}} \cap L^{\infty}}} d \tau$.

We want to study now the following problem:

$$
\left\{\begin{array}{l}
\partial_{t} q+u \cdot \nabla q+\alpha q=F, \\
q_{t=0}=q_{0}
\end{array}\right.
$$

Above $q$ is the unknown function. We assume that $F \in L^{r}\left(0, T ; B_{p, r}^{s}\right)$, that $v$ is time dependent vector-fields with coefficients in $L^{1}\left(0, T ; B_{p_{1}, 1}^{\frac{N}{p_{1}}+1}\right)$ and $\alpha>0$ a constant. 
Proposition 3.6 Let $1 \leq p_{1} \leq p \leq+\infty, r \in[1,+\infty]$ and $s \in \mathbb{R}$ be such that:

$$
-N \min \left(\frac{1}{p_{1}}, \frac{1}{p^{\prime}}\right)<s<1+\frac{N}{p_{1}} .
$$

There exists a constant $C$ depending only on $N, p, p_{1}, r$ and $s$ such that for all $a \in$ $L^{\infty}\left([0, T], B_{p, r}^{\sigma}\right)$ of $(\mathcal{H})$ with initial data $a_{0}$ in $B_{p, r}^{s}$ and $g \in L^{1}\left([0, T], B_{p, r}^{s}\right)$, we have for a.e $t \in[0, T]:$

$$
\|q\|_{\widetilde{L}_{t}^{\infty}\left(B_{p, r}^{s}\right)}+\|q\|_{\widetilde{L}_{t}^{1}\left(B_{p, r}^{s}\right)} \leq e^{C U(t)}\left(\left\|q_{0}\right\|_{B_{p, r}^{s}}+\int_{0}^{t} e^{-C U(\tau)}\|F(\tau)\|_{B_{p, r}^{s}} d \tau\right)
$$

with: $U(t)=\int_{0}^{t}\|\nabla u(\tau)\|_{B_{p_{1}, \infty}^{\frac{N}{p_{1}} \cap L^{\infty}}} d \tau$.

Proof: Applying $\Delta_{l}$ to $(\mathcal{H})$ yields:

$$
\partial_{t} \Delta_{l} q+u \cdot \nabla \Delta_{l} q+\alpha \Delta_{l} q=R_{l}+\Delta_{l} F
$$

with $R_{l}=\left[u \cdot \nabla, \Delta_{l}\right] q$. Multiplying by $\Delta_{l} a\left|\Delta_{l} a\right|^{p-2}$ then performing a time integration, we easily get:

$$
\begin{array}{r}
\left\|\Delta_{l} q(t)\right\|_{L^{p}}+\alpha \int_{0}^{t}\left\|\Delta_{l} q(s)\right\|_{L^{p}} d s \leq\left\|\Delta_{l} q_{0}\right\|_{L^{p}}+\int_{0}^{t}\left(\left\|R_{l}\right\|_{L^{p}}+\frac{1}{p}\|\operatorname{div} u\|_{L^{\infty}}\left\|\Delta_{l} q\right\|_{L^{p}}\right. \\
\left.+\left\|\Delta_{l} F\right\|_{L^{p}}\right) d \tau .
\end{array}
$$

Next the term $\left\|R_{l}\right\|_{L^{p}}$ may be bounded according to lemma 2 in appendix. We get then:

$$
\|q\|_{\widetilde{L}_{t}^{\infty}\left(B_{p, r}^{s}\right)}+\alpha\|q\|_{\widetilde{L}_{t}^{1}\left(B_{p, r}^{s}\right)} d s \leq\left\|\Delta_{l} q_{0}\right\|_{B_{p, r}^{s}}+\int_{0}^{t}\left(\|F(\tau)\|_{B_{p, r}^{s}}+C U^{\prime}(\tau)\|q\|_{\widetilde{L}_{t}^{\infty}\left(B_{p, r}^{s}\right)}\right) d \tau .
$$

We end up with Grönwall lemma by letting $X(t)=\|q\|_{\widetilde{L}_{t}^{\infty}\left(B_{p, r}^{s}\right)}+\alpha\|q\|_{\widetilde{L}_{t}^{1}\left(B_{p, r}^{s}\right)}$.

\section{The proof of theorem 1.1}

\subsection{Strategy of the proof}

To improve the results of Danchin in [14], Charve and Danchin in [7] and Chen et al in [11], it is crucial to kill the coupling between the velocity and the pressure which exists in these works. To achieve it, we need to include the pressure term in the study of the linearized equation of the momentum equation as in [21]. For that, we will try to express the gradient of the pressure as a Laplacian term, so we have to solve:

$$
\Delta v=\nabla P(\rho) .
$$

Let $\mathcal{E}$ be the fundamental solution of the Laplace operator. We will set in the sequel: $v=\nabla \mathcal{E} *(P(\rho)-P(\bar{\rho}))=\nabla(\mathcal{E} *[P(\rho)-P(\bar{\rho})])$ (* here means the operator of convolution). We verify next that:

$$
\nabla \operatorname{div} v=\nabla \Delta(\mathcal{E} *[P(\rho)-P(\bar{\rho})])=\Delta \nabla(\mathcal{E} *[P(\rho)-P(\bar{\rho})])=\Delta v=\nabla P(\rho) .
$$


By this way we can now rewrite the momentum equation of (1.3) as:

$$
\partial_{t} u+u \cdot \nabla u-\frac{\mu}{\rho} \Delta\left(u-\frac{1}{\nu} v\right)-\frac{\lambda+\mu}{\rho} \nabla \operatorname{div}\left(u-\frac{1}{\nu} v\right)=f,
$$

with $\nu=2 \mu+\lambda$. We now want to calculate $\partial_{t} v$, by the transport equation we get:

$$
\partial_{t} v=\nabla \mathcal{E} * \partial_{t} P(\rho)=-\nabla \mathcal{E} *\left(P^{\prime}(\rho) \operatorname{div}(\rho u)\right) .
$$

Notation 2 To simplify the notation, we will note in the sequel

$$
\nabla \mathcal{E} *\left(P^{\prime}(\rho) \operatorname{div}(\rho u)\right)=\nabla(\Delta)^{-1}\left(P^{\prime}(\rho) \operatorname{div}(\rho u)\right) .
$$

Finally we can now rewrite the system (1.3) as follows:

$$
\left\{\begin{aligned}
\partial_{t} q+\left(v_{1}+\frac{1}{\nu} v\right) \cdot \nabla q & +\frac{1}{\nu} P^{\prime}(1) q=-(1+q) \operatorname{div} v_{1} \\
& -\frac{1}{\nu}\left(P(\rho)-P(1)-P^{\prime}(1)\right)-\frac{1}{\nu} q(P(\rho)-P(1)) \\
\partial_{t} v_{1}-\frac{1}{1+q} \mathcal{A} v_{1}= & f-u \cdot \nabla u+\frac{1}{\nu} \nabla(\Delta)^{-1}\left(P^{\prime}(\rho) \operatorname{div}(\rho u)\right) \\
q_{/ t=0}=a_{0},\left(v_{1}\right)_{/ t}=0 & =\left(v_{1}\right)_{0}
\end{aligned}\right.
$$

where $v_{1}=u-\frac{1}{\nu} v$ is called the effective velocity. In the sequel we will study this system by exhibiting some uniform bounds in Besov spaces on $\left(q, v_{1}\right)$. The advantage of the system (4.13) is that we have canceled out the coupling between $v_{1}$ and a term of pressure. Indeed in the works [7] and [11], the pressure was included in the study of the linear system, thus entailing a coupling between the density and the velocity. In particular it was impossible to prescribe different index of integration in Besov spaces for the velocity and the density.

\subsection{A linear model with convection}

In this section, we will explain how we treat the low frequency regime by following the approach of Charve and Danchin in [7]. In low frequencies, the first order terms predominate and the viscous term $\Delta u$ may (almost) be neglected so that (1.1) has to be treated by means of hyperbolic energy methods. It means that we can only work in spaces constructed on $L^{2}$. Moreover in the case of low frequencies the effective velocity is not a suitable unknown in the sense that is less regular than $u$ as $(\Delta)^{-1} \nabla P(\rho)$ is not very regular in low frequencies (indeed the index of regularity for the Besov space is to high) . It is better in this case to work with $u$. Namely the first idea would be to study the linear system associated to (1.1):

$$
\left\{\begin{array}{l}
\partial_{t} q+\operatorname{div} u=F^{\prime}, \\
\partial_{t} u-\mu \Delta u-\lambda \nabla \operatorname{div} u+\nabla q=G^{\prime} .
\end{array}\right.
$$

This system has been studied by D. Hoff and K. Zumbrun in [31]. There, they investigate the decay estimates, and exhibit the parabolic smoothing effect on $u$ and on the low frequencies of $q$, and a damping effect on the high frequencies of $q$. 
The problem is that if we focus on this linear system, it appears impossible to control the term of convection $u \cdot \nabla q$ which is one derivative less regular than $q$. However in low frequencies the Green matrix of the linearized system behaves as the heat kernel ( see [11]), the terms $v \cdot \nabla q$ and $v \cdot \nabla u$ can be handled as the perturbation terms. We study then the following system:

$$
\left\{\begin{array}{l}
\partial_{t} q+\operatorname{div} u=F-v \cdot \nabla q, \\
\partial_{t} u-\mu \Delta u-\lambda \nabla \operatorname{div} u+\nabla q=G-v \cdot \nabla u .
\end{array}\right.
$$

Proposition 4.7 Let $(q, u)$ a solution of $(L H)^{\prime}$, let $s \in \mathbb{R}$. The following estimate holds:

$$
\begin{array}{r}
\left\|(q, u)_{B F}\right\|_{\widetilde{L}^{\infty}\left(B_{2,1}^{s}\right)}+\left\|(q, u)_{B F}\right\|_{\widetilde{L}^{1}\left(B_{2,1}^{s+2}\right)} \leq\left\|\left(q_{0}, u_{0}\right)_{B F}\right\|_{B_{2,1}^{s}}+\left\|(F, G)_{B F}\right\|_{\widetilde{L}^{1}\left(B_{2,1}^{s}\right)} \\
+\left\|(v \cdot \nabla q, v \cdot \nabla u)_{B F}\right\|_{\widetilde{L}^{1}\left(B_{2,1}^{s}\right)} .
\end{array}
$$

Proof: In this case for $j \leq 0$, in terms of Green matrix (see [11]), the solution of $(L H)^{\prime}$ can be expressed as:

$$
\left(\begin{array}{c}
\Delta_{j} q(t) \\
\Delta_{j} u(t)
\end{array}\right)=W(t)\left(\begin{array}{c}
\Delta_{j} q_{0} \\
\Delta_{j} u_{0}
\end{array}\right)+\int_{0}^{t} W(t-s)\left(\begin{array}{c}
\Delta_{j} F(s)-\Delta_{j}(v \cdot \nabla q)(s) \\
\Delta_{j} G(s)-\Delta_{j}(v \cdot \nabla u)(s)
\end{array}\right) d s
$$

with $W$ the Green matrix. From proposition 4.4 in [11] and Young's inequality we obtain the result.

\subsection{Proof of the existence}

\section{Construction of approximate solutions}

We use a standard scheme:

1. We smooth out the data and get a sequence of local solutions $\left(q^{n}, u^{n}\right)_{n \in \mathbb{N}}$ on $\left[0, T_{n}\right]$ to $(1.3)$ on $\mathbb{R}$ by using the result of [17].

2. We prove uniform estimates on $\left(q^{n}, v_{1}^{n}\right)$ in high frequencies and on $\left(q^{n}, u^{n}\right)$ in low frequencies on $\left[0, T_{n}\right]$ and we deduce that $T_{n}=+\infty$.

3. We use compactness to prove that the sequence $\left(q^{n}, u^{n}\right)$ converges, up to extraction, to a solution of (1.1).

\section{First step}

We smooth out the data as follows:

$$
q_{0}^{n}=S_{n} q_{0}, \quad u_{0}^{n}=S_{n} u_{0} \quad \text { and } \quad f^{n}=S_{n} f .
$$

Note that we have:

$$
\forall l \in \mathbb{Z}, \quad\left\|\Delta_{l} q_{0}^{n}\right\|_{L^{p}} \leq\left\|\Delta_{l} q_{0}\right\|_{L^{p}} \quad \text { and } \quad\left\|q_{0}^{n}\right\|_{B_{p, 1}^{\frac{N}{p}} \cap B_{p, 1}^{\frac{N}{p}-1}} \leq\left\|q_{0}\right\|_{B_{p, 1}^{\frac{N}{p}} \cap B_{p, 1}^{\frac{N}{p}-1}},
$$

and similar properties for $u_{0}^{n}$ and $f^{n}$, a fact which will be used repeatedly during the next steps. Now, according [17], one can solve (1.3) with the smooth data $\left(q_{0}^{n}, u_{0}^{n}, f^{n}\right)$. We get a solution $\left(q^{n}, u^{n}\right)$ such that:

$$
q^{n} \in \widetilde{C}\left(\left[0, T_{n}\right], B_{2,1}^{N} \cap B_{2,1}^{\frac{N}{2}-1}\right) u^{n} \in \widetilde{C}\left(\left[0, T_{n}\right],, B_{2,1}^{\frac{N}{2}-1}\right) \cap \widetilde{L}^{1}\left(\left[0, T_{n}\right],, B_{2,1}^{\frac{N}{2}+1}\right) .
$$




\section{Uniform bounds}

We set now

$$
v_{n}=\nabla\left(\mathcal{E} *\left[P\left(\rho^{n}\right)-P(1)\right]\right) \quad \text { with } \quad \operatorname{div} v^{n}=P\left(\rho^{n}\right)-P(1) \quad \text { and } \quad v_{1}^{n}=u^{n}-\frac{1}{\nu} v^{n},
$$

with $\mathcal{E}$ the fundamental solution of the Laplace operator and $\nu=\lambda+2 \mu$. In the sequel we will note $g\left(q^{n}\right)=P\left(\rho^{n}\right)-P(1)$ where $g$ is a regular function.

In this part, we aim at getting uniform estimates on $\left(q_{H F}^{n},\left(v_{1}\right)_{H F}^{n}\right)$ in high frequencies and on $\left(q_{B F}^{n}, u_{B F}^{n}\right)$ in low frequencies in the following space $E^{\prime}$ and $F^{\prime}$ :

$$
\begin{aligned}
& E^{\prime}=\left(\widetilde{L}^{\infty}\left(B_{p, 1}^{\frac{N}{p}}\right) \cap \widetilde{L}^{1}\left(B_{p, 1}^{\frac{N}{p}}\right)\right) \times\left(\widetilde{L}^{\infty}\left(B_{p_{1}, 1}^{\frac{N}{p_{1}}-1}+B_{p, 1}^{\frac{N}{p}}\right)+\widetilde{L}^{1}\left(B_{p_{1}, 1}^{\frac{N}{p_{1}}+1}+B_{p, 1}^{\frac{N}{p}+2}\right)\right) . \\
& F^{\prime}=\left(\widetilde{L}^{\infty}\left(B_{2,1}^{\frac{N}{2}-1}\right) \cap \widetilde{L}^{1}\left(B_{2,1}^{\frac{N}{2}+1}\right)\right) \times\left(\widetilde{L}^{\infty}\left(B_{2,1}^{\frac{N}{2}-1}\right)+\widetilde{L}^{1}\left(B_{2,1}^{\frac{N}{2}+1}\right)\right) .
\end{aligned}
$$

More precisely we will obtain uniform estimates on $\left(q^{n}, u^{n}\right)$ in $E$ and on $\left(q^{n}, v_{1}^{n}\right)$ in $F$ whith:

$$
\begin{array}{r}
E=\left(\widetilde{L}^{\infty}\left(\widetilde{B}_{2, p, 1}^{\frac{N}{2}-1, \frac{N}{p}}\right) \cap \widetilde{L}^{1}\left(\widetilde{B}_{2, p, 1}^{\frac{N}{2}+1, \frac{N}{p}}\right)\right) \times\left(\widetilde{L}^{\infty}\left(\widetilde{B}_{2, p, 1}^{\frac{N}{2}-1, \frac{N}{p}}+\widetilde{B}_{2, p_{1}, 1}^{\frac{N}{2}-1, \frac{N}{p_{1}}-1}\right)\right. \\
\left.\cap \widetilde{L}^{1}\left(\widetilde{B}_{2, p, 1}^{\frac{N}{2}+1, \frac{N}{p}+1}\right)\right) . \\
\begin{aligned}
F=\widetilde{L}^{\infty}\left(\widetilde{B}_{2, p, 1}^{\frac{N}{2}-1, \frac{N}{p}}\right) \cap \widetilde{L}^{1}\left(\widetilde{B}_{2, p, 1}^{\frac{N}{2}+1, \frac{N}{p}}\right) \times\left(\widetilde{L}^{\infty}\left(\widetilde{B}_{2, p, 1}^{\frac{N}{2}, \frac{N}{p}}+\widetilde{B}_{2, p_{1}, 1}^{\frac{N}{2}, \frac{N}{1}-1}\right)\right. \\
\left.\cap \widetilde{L}^{1}\left(\widetilde{B}_{2, p, 1}^{\frac{N}{2}+2, \frac{N}{p}+2}+\widetilde{B}_{2, p_{1}, 1}^{\frac{N}{2}+2, \frac{N}{p_{1}}+1}\right)\right) .
\end{aligned}
\end{array}
$$

We will work finally in the space $H$ with:

$$
(q, u) \in H_{T} \Leftrightarrow(q, u)_{B F} \in E_{T}^{\prime} \text { and }\left(q, v_{1}\right)_{H F} \in F_{T}^{\prime} .
$$

Here when we write $E_{T}^{\prime}$ or $F_{T}^{\prime}$, we means that we consider $E^{\prime}$ or $F^{\prime}$ locally in time on the interval $[0, T]$. We have then: $\|(q, u)\|_{H}=\left\|(q, u)_{B F}\right\|_{E^{\prime}}+\left\|\left(q, v_{1}\right)_{H F}\right\|_{F^{\prime}}$. We can now check that $\left(q^{n}, v_{1}^{n}\right)$ satisfies the following system:

$$
\left\{\begin{array}{l}
\partial_{t} q^{n}+u^{n} \cdot \nabla q^{n}+\frac{P^{\prime}(1)}{\nu} q^{n}=F_{1}^{n}, \\
\partial_{t} v_{1}^{n}-\mathcal{A} v_{1}^{n}=G_{1}^{n}+f, \\
q_{0}^{n}=q_{0},\left(v_{1}^{n}\right)_{/ t=0}=u_{0}^{n}-\frac{1}{\nu} v_{0}^{n} .
\end{array}\right.
$$

which is a transport equation and a heat equation. Here we have:

$$
\begin{aligned}
& F_{1}^{n}=-\left(1+q^{n}\right) \operatorname{div} v_{1}^{n}-\frac{1}{\nu}\left(P\left(1+q^{n}\right)-P(1)-P^{\prime}(1) q^{n}\right)-\frac{1}{\nu} q^{n}\left(P\left(1+q^{n}\right)-P(1)\right), \\
& G_{1}^{n}=\left(\frac{1}{1+q^{n}}-1\right) \mathcal{A} v_{1}^{n}-u^{n} \cdot \nabla u^{n}+\frac{1}{\nu} \nabla(\Delta)^{-1}\left(P^{\prime}\left(\rho^{n}\right) \operatorname{div}\left(\rho^{n} u^{n}\right)\right) .
\end{aligned}
$$

Moreover $\left(q^{n}, u^{n}\right)_{n \in \mathbb{N}}$ is the solution of the following system:

$$
\left\{\begin{array}{l}
\partial_{t} q^{n}+u^{n} \cdot \nabla q^{n}+\operatorname{div} u^{n}=F^{n} \\
\partial_{t} u^{n}+u^{n} \cdot \nabla u^{n}-\mathcal{A} u^{n}+P^{\prime}(1) \nabla q^{n}=G^{n}+f^{n} \\
\left(q^{n}, u^{n}\right)_{/ t=0}=\left(q_{0}^{n}, u_{0}^{n}\right),
\end{array}\right.
$$


with:

$$
\begin{aligned}
F^{n} & =-q^{n} \operatorname{div} u^{n} \\
G^{n} & =-\frac{q^{n}}{1+q^{n}} \mathcal{A} u^{n}+\left(P^{\prime}(1)-P^{\prime}\left(1+q^{n}\right)\right) \nabla q^{n} .
\end{aligned}
$$

Let us set:

$$
\begin{aligned}
& E(q, u)=\|q\|_{\widetilde{L}^{\infty}\left(\widetilde{B}_{2, p, 1}^{\frac{N}{2}-1, \frac{N}{p}}\right)}+\|u\|_{\widetilde{L}^{\infty}\left(\widetilde{B}_{2, p_{1}, 1}^{\frac{N}{2}-1, \frac{N}{p_{1}}-1}+\widetilde{B}_{2, p, 1}^{\frac{N}{2}-1, \frac{N}{p}}\right)}+\|q\|_{L^{1}\left(\widetilde{B}_{2, p, 1}^{\frac{N}{2}+1, \frac{N}{p}}\right)} \\
& +\|u\|_{L^{1}\left(\widetilde{B}_{2, p, 1}^{\frac{N}{2}+1, \frac{N}{p}+1}\right)}, \\
& E_{1}(q, u)=\|q\|_{\widetilde{L}^{\infty}\left(B_{2,1}^{\frac{N}{2}-1}\right)}+\|u\|_{\widetilde{L}^{\infty}\left(B_{2,1}^{\frac{N}{2}-1}\right)}+\|q\|_{L^{1}\left(B_{2,1}^{\frac{N}{2}+1}\right)}+\|u\|_{L^{1}\left(B_{2,1}^{\frac{N}{2}+1}\right)} . \\
& E_{2}(q, u)=\|q\|_{\widetilde{L}^{\infty}\left(B_{p, 1}^{\frac{N}{p}}\right)}+\|u\|_{\widetilde{L}^{\infty}\left(B_{p_{1}, 1}^{\frac{N}{p_{1}}-1}+B_{p, 1}^{\frac{N}{p}}\right)}+\|q\|_{L^{1}\left(B_{p, 1}^{\frac{N}{p}}\right)} \\
& +\|u\|_{L^{1}\left(B_{p_{1}, 1}^{\frac{N}{p_{1}}+1}+B_{p, 1}^{\frac{N}{p}+2}\right)} .
\end{aligned}
$$

In the sequel we will work always on the interval $\left[0, T_{n}\right]$, but for simplifying the notation we do not mention the time $T_{n}$ in the Lebesgue spaces. One can now apply the proposition 2.4 to our system to obtain uniform bounds. We have then in high frequencies to control $\left(v_{1}^{n}, q^{n}\right)$ and in low frequencies for $\left(q^{n}, u^{n}\right)$ :

$$
\begin{aligned}
E_{2}\left(\left(q^{n}, v_{1}^{n}\right)_{H F}\right) \leq C\left(\left\|\left(q_{0}\right)_{H F}\right\|_{B_{p, 1}^{\frac{N}{p}-1}+B_{p, 1}^{\frac{N}{p}}}+\left\|\left(u_{0}\right)_{H F}\right\|_{B_{p_{1}, 1}^{\frac{N}{p_{1}}-1}}\right. \\
\left.+\left\|\left(F_{1}^{n}\right)_{H F}\right\|_{L^{1}\left(B_{p, 1}^{\frac{N}{p}}\right)}+\left\|G_{1}^{n}\right\|_{L^{1}\left(B_{p_{1}, 1}^{\frac{N}{p_{1}}-1}+B_{p, 1}^{\frac{N}{p}}\right)}\right),
\end{aligned}
$$

and

$$
\begin{aligned}
E_{1}\left(\left(q^{n}, u^{n}\right)_{B F}\right) \leq C\left(\left\|\left(q_{0}\right)_{B F}\right\|_{B_{2,1}^{\frac{N}{2}}-1}+\left\|\left(u_{0}\right)_{B F}\right\|_{B_{2,1}^{\frac{N}{2}}-1}\right. & \\
& \left.+\left\|\left(F^{n}\right)_{B F}\right\|_{L^{1}\left(B_{2,1}^{\frac{N}{2}-1}\right)}+\left\|G^{n}\right\|_{L^{1}\left(B_{2,1}^{\frac{N}{2}-1}\right)}\right),
\end{aligned}
$$

Therefore, it is only a matter of proving appropriate estimates for $F_{1}^{n}, G_{1}^{n} F^{n}$ and $G^{n}$ by using properties of continuity on the paraproduct and proposition 2.4, 4.7.

We begin by estimating $\left\|\left(F_{1}^{n}\right)_{H F}\right\|_{\widetilde{L}^{1}\left(B_{p, 1}^{\frac{N}{p}}\right)}$ and $\left\|\left(G_{1}^{n}\right)_{H F}\right\|_{\widetilde{L}^{1}\left(B_{p_{1}, 1}^{\frac{N}{p_{1}}-1}+B_{p, 1}^{\frac{N}{p}}\right)}$, we have to use proposition 2.2 and proposition 6.8 and the fact that by interpolation $\operatorname{div} v_{1}^{n}$ is in $\widetilde{L}^{1}\left(\widetilde{B}_{2, p, 1}^{\frac{N}{2}+1, \frac{N}{p}}\right)$ because $\widetilde{L}^{1}\left(\widetilde{B}_{2, p, 1}^{\frac{N}{2}+1, \frac{N}{p}+1}+\widetilde{B}_{2, p_{1}, 1}^{\frac{N}{2}+1, \frac{N}{p_{1}}}\right) \hookrightarrow \widetilde{L}^{1}\left(\widetilde{B}_{2, p, 1}^{\frac{N}{2}+1, \frac{N}{p}}\right)$ as $p_{1} \leq p$ :

$$
\begin{gathered}
\left\|\left(\left(1+q^{n}\right) \operatorname{div} v_{1}^{n}\right)_{H F}\right\|_{\widetilde{L}^{1}\left(B_{p, 1}^{\frac{N}{p}}\right)} \leq\left\|\operatorname{div} v_{1}^{n}\right\|_{\widetilde{L}^{1}\left(\widetilde{B}_{2, p, 1}^{\frac{N}{2}+1, \frac{N}{p}}\right)}+\|q\|_{L^{\infty}\left(L^{\infty}\right)}\left\|\operatorname{div} v_{1}^{n}\right\|_{\widetilde{L}^{1}\left(\widetilde{B}_{2, p, 1}^{\frac{N}{2}+1, \frac{N}{p}}\right)} \\
+\left\|\operatorname{div} v_{1}^{n}\right\|_{L^{1}\left(L^{\infty}\right)}\left\|q^{n}\right\|_{\widetilde{L}^{\infty}\left(\widetilde{B}_{2, p, 1}^{\frac{N}{2}-1, \frac{N}{p}}\right)} \\
\left\|\left[\left(P\left(1+q^{n}\right)-P(1)-P^{\prime}(1) q^{n}\right)\right]_{H F}\right\|_{\widetilde{L}^{1}\left(B_{p, 1}^{\frac{N}{p}}\right)} \leq C\left\|q^{n}\right\|_{\widetilde{L}^{2}\left(\widetilde{B}_{2, p, 1}^{\left.\frac{N}{2}, \frac{N}{p}\right)}\right.}^{2} \\
\left\|\left[q^{n}\left(P\left(1+q^{n}\right)-P(1)\right)\right]_{H F}\right\|_{\widetilde{L}^{1}\left(B_{p, 1}^{\frac{N}{p}}\right)} \leq C\left\|q^{n}\right\|_{\widetilde{L}^{2}\left(\widetilde{B}_{2, p, 1}^{\left.\frac{N}{2}, \frac{N}{p}\right)}\right.}^{2}
\end{gathered}
$$


Next we have to treat the term $\left[\frac{q^{n}}{1+q^{n}} \mathcal{A} v_{1}^{n}\right]_{H F}$ in $\widetilde{L}^{1}\left(B_{p_{1}, 1}^{\frac{N}{p_{1}}-1}+B_{p, 1}^{\frac{N}{p}}\right)$, where we can split $\mathcal{A} v_{1}^{n}$ on the form:

$$
v_{1}^{n}=h^{n}+g^{n}
$$

with: $h^{n} \in \widetilde{L}^{\infty}\left(\widetilde{B}_{2, p_{1}, 1}^{\frac{N}{2}-1, \frac{N}{p_{1}}-1}\right) \cap \widetilde{L}^{1}\left(\widetilde{B}_{2, p_{1}, 1}^{\frac{N}{2}+2, \frac{N}{p_{1}}+1}\right)$ and $g^{n} \in \widetilde{L}^{\infty}\left(\widetilde{B}_{2, p, 1}^{\frac{N}{2}, \frac{N}{p}}\right) \cap \widetilde{L}^{1}\left(\widetilde{B}_{2, p, 1}^{\frac{N}{2}+2, \frac{N}{p}+2}\right)$. We obtain then by proposition 6.8 :

$$
\begin{gathered}
\left\|\left[\frac{q^{n}}{1+q^{n}} \mathcal{A} g^{n}\right]_{H F}\right\|_{\widetilde{L}^{1}\left(B_{p, 1}^{\frac{N}{p}}\right)} \leq\left\|T_{\frac{q^{n}}{1+q^{n}}} \mathcal{A} g^{n}\right\|_{\widetilde{L}^{1}\left(\widetilde{B}_{2, p, 1}^{\frac{N}{2}, \frac{N}{p}}\right)}+\left\|T_{\mathcal{A} g^{n}} \frac{q^{n}}{1+q^{n}}\right\|_{\widetilde{L}^{1}\left(\widetilde{B}_{2, p, 1}^{\frac{N}{2}-1, \frac{N}{p}}\right)} \\
+\left\|R\left(\mathcal{A} g^{n}, \frac{q^{n}}{1+q^{n}}\right)\right\|_{\widetilde{L}^{1}\left(\widetilde{B}_{2, p, 1}^{\frac{N}{2}, \frac{N}{p}}\right)}, \\
\leq C\left\|q^{n}\right\|_{\widetilde{L}_{\widetilde{L}_{(}\left(\widetilde{B}_{2, p, 1}^{\frac{N}{2}-1, \frac{N}{p}}\right)}\left\|\mathcal{A} g^{n}\right\|_{\widetilde{L}^{1}\left(\widetilde{B}_{2, p, 1}^{\frac{N}{2}, \frac{N}{p}}\right)} \cdot}
\end{gathered}
$$

Next we have to use proposition 6.8 to treat the term $T_{\mathcal{A} h^{n}}\left(\frac{1}{1+q^{n}}-1\right)$ and $R\left(\mathcal{A} h^{n}, \frac{1}{1+q^{n}}-1\right)$ when $p_{1}>N$, we have then:

$$
\left\|T_{\mathcal{A} h^{n}} \frac{q^{n}}{1+q^{n}}\right\|_{\widetilde{L}^{1}\left(\widetilde{B}_{2, p_{1}, 1}^{\frac{N}{2}, \frac{N}{p_{1}}-1}\right)} \leq\left\|\mathcal{A} h^{n}\right\|_{\widetilde{L}^{1}\left(\widetilde{B}_{2, p_{1}, 1}^{\frac{N}{2}, \frac{N}{p_{1}}-1}\right)}\left\|\frac{q^{n}}{1+q^{n}}\right\|_{\widetilde{L}^{\infty}\left(\widetilde{B}_{2, p, 1}^{\frac{N}{2}-1, \frac{N}{p}}\right)},
$$

where following the proposition 6.8 , we have chosen $p=2, q=p_{1}$, and as $p \geq p_{1}$ we have $\frac{1}{\lambda^{\prime}}=\frac{1}{p_{1}}-\frac{1}{p}$ and $\lambda=+\infty$. It means that: $\frac{N}{p_{1}}-1 \leq \frac{N}{p}$ (what is assumed) and $2 \leq \lambda^{\prime}$ if $2 \geq \frac{p_{1} p}{p-p_{1}}$. We need then the following assumption:

$$
2 \geq \frac{p_{1} p}{p-p_{1}} \text { and } \quad \frac{N}{p_{1}}-1 \leq \frac{N}{p}
$$

Next we have as $\frac{N}{p_{1}}+\frac{N}{p}-1>0$ by proposition 6.8 for the remainder term on the high frequencies:

$$
\left\|\left[R\left(\mathcal{A} h^{n},\left(\frac{1}{1+q^{n}}-1\right)\right)\right]_{H F}\right\|_{\widetilde{L}^{1}\left(B_{p_{1}, 1}^{\frac{N}{p_{1}}-1}\right)} \leq\left\|h^{n}\right\|_{\widetilde{L}^{1}\left(B_{2, p_{1}, 1}^{\frac{N}{2}-1, \frac{N}{p_{1}}-1}\right)}\left\|\frac{1}{1+q^{n}}-1\right\|_{\widetilde{L}^{\infty}\left(B_{2, p, 1}^{\frac{N}{2}-1, \frac{N}{p}}\right)} .
$$

We have seen that we need the following condition to treat this term:

$$
\frac{N}{p_{1}}+\frac{N}{p}-1>0
$$

Easily we have by proposition 6.8 as $\widetilde{L}^{\infty}\left(\widetilde{B}_{2, p, 1}^{\frac{N}{2}-1, \frac{N}{p}}\right) \hookrightarrow L^{\infty}$ :

$$
\left.\| T_{\frac{q^{n}}{1+q^{n}}-1} \mathcal{A} h^{n}\right]_{H F}\left\|_{\widetilde{L}^{1}\left(\widetilde{B}_{p_{1}, 1}^{\frac{N}{p_{1}}-1}\right)} \leq\right\| \mathcal{A} h^{n}\left\|_{\widetilde{L}^{1}\left(\widetilde{B}_{2, p_{1}, 1}^{\frac{N}{2}-1, \frac{N}{p_{1}}-1}\right)}\right\| \frac{q^{n}}{1+q^{n}} \|_{\widetilde{L}^{\infty}\left(\widetilde{B}_{2, p, 1}^{\frac{N}{2}-1, \frac{N}{p}}\right)} .
$$

We now treat the term $u^{n} \cdot \nabla u^{n}$ and we have as $u^{n} \in E$, it exists $h_{1}^{n}$ and $g_{1}^{n}$ such that $u^{n}=g_{1}^{n}+h_{1}^{n}$ with $h_{1}^{n} \in \widetilde{L}^{\infty}\left(\widetilde{B}_{2, p, 1}^{\frac{N}{2}-1, \frac{N}{p}}\right) \cap \widetilde{L}^{1}\left(\widetilde{B}_{2, p, 1}^{\frac{N}{2}+1, \frac{N}{p}+1}\right)$ and $g_{1}^{n} \in \widetilde{L}^{\infty}\left(\widetilde{B}_{2, p_{1}, 1}^{\frac{N}{2}-1, \frac{N}{p_{1}}-1}\right) \cap$ 
$\widetilde{L}^{1}\left(\widetilde{B}_{2, p, 1}^{\frac{N}{2}+1, \frac{N}{p}+1}\right)$. We have then by proposition 6.8 :

$$
\begin{aligned}
& \left\|\left(h_{1}^{n} \cdot \nabla h_{1}^{n}\right)_{H F}\right\|_{\widetilde{L}^{1}\left(B_{p, 1}^{\frac{N}{p}}\right)} \leq\left\|T_{h_{1}^{n}} \nabla h_{1}^{n}\right\|_{\widetilde{L}^{1}\left(\widetilde{B}_{2, p, 1}^{\left.\frac{N}{2}, \frac{N}{p}\right)}\right.}+\left\|T_{\nabla h_{1}^{n}} h_{1}^{n}\right\|_{\widetilde{L}^{1}\left(\widetilde{B}_{2, p, 1}^{\frac{N}{2}-1, \frac{N}{p}}\right)} \\
& +\left\|R\left(\nabla h_{1}^{n}, h_{1}^{n}\right)\right\|_{\widetilde{L}^{1}\left(\widetilde{B}_{2, p, 1}^{\left.\frac{N}{2}, \frac{N}{p}\right)}\right.}, \\
& \leq\left\|h_{1}^{n}\right\|_{\widetilde{L}^{1}\left(\widetilde{B}_{2, p, 1}^{\frac{N}{2}+1, \frac{N}{p}+1}\right)}\left\|h_{1}^{n}\right\|_{\widetilde{L}^{\infty}\left(\widetilde{B}_{2, p, 1}^{\frac{N}{2}-1, \frac{N}{p}}\right)} .
\end{aligned}
$$

Next we have to treat the term $T_{g_{1}^{n}} \nabla g_{1}^{n}$ by using the proposition 6.8 with $\frac{1}{\lambda^{\prime}}=\frac{1}{p_{1}}-\frac{1}{p}$, $2 \leq \lambda^{\prime}$ and $\frac{N}{p_{1}}-1 \leq \frac{N}{p}$ then:

$$
\left\|T_{g_{1}^{n}} \nabla g_{1}^{n}\right\|_{\widetilde{L}^{1}\left(\widetilde{B}_{2, p_{1}, 1}^{\frac{N}{2}-1, \frac{N}{p_{1}}-1}\right)} \leq\left\|g_{1}^{n}\right\|_{\widetilde{L}^{\infty}\left(\widetilde{B}_{2, p_{1}, 1}^{\frac{N}{2}-1, \frac{N}{p_{1}}-1}\right)}\left\|\nabla g_{1}^{n}\right\|_{\widetilde{L}^{1}\left(\widetilde{B}_{2, p, 1}^{\frac{N}{2}, \frac{N}{p}}\right)} .
$$

We have seen that we need the hypothesis:

$$
\frac{N}{p_{1}}-1 \leq \frac{N}{p} \text { and } 2 \leq \frac{p_{1} p}{p-p_{1}}
$$

Easily we have as $\widetilde{B}_{2, p, 1}^{\frac{N}{2}, \frac{N}{p}} \hookrightarrow L^{\infty}$ by proposition 6.8 :

$$
\left\|T_{\nabla g_{1}^{n}} g_{1}^{n}\right\|_{\widetilde{L}^{1}\left(\widetilde{B}_{2, p_{1}, 1}^{\frac{N}{2}-1, \frac{N}{p_{1}}-1}\right)} \leq C\left\|g_{1}^{n}\right\|_{\widetilde{L}^{\infty}\left(\widetilde{B}_{2, p_{1}, 1}^{\frac{N}{2}-1, \frac{N}{p_{1}}-1}\right)}\left\|\nabla g_{1}^{n}\right\|_{\widetilde{L}^{1}\left(\widetilde{B}_{2, p, 1}^{\frac{N}{2}, \frac{N}{p}}\right)} .
$$

To finish with the term $g_{1}^{n} \nabla g_{1}^{n}$, we have to treat the term $\left(R\left(g_{1}^{n}, \nabla g_{1}^{n}\right)\right)_{H F}$. By proposition 6.8 , as $\frac{N}{p}+\frac{N}{p_{1}}-1>0$ we have:

$$
\left\|\left(R\left(g_{1}^{n}, \nabla g_{1}^{n}\right)\right)_{H F}\right\|_{\widetilde{L}^{1}\left(B_{p_{1}, 1}^{\frac{N}{p_{1}}-1}\right)} \leq C\left\|g_{1}^{n}\right\|_{\widetilde{L}^{\infty}\left(\widetilde{B}_{2, p_{1}, 1}^{\frac{N}{2}-1, \frac{N}{p_{1}}-1}\right)}\left\|\nabla g_{1}^{n}\right\|_{\widetilde{L}^{1}\left(\widetilde{B}_{2, p, 1}^{\frac{N}{2}, \frac{N}{p}}\right)} .
$$

We have seen that we need the condition:

$$
\frac{N}{p_{1}}-1+\frac{N}{p} \geq 0
$$

From the previous inequalities, we have obtained:

$$
\left\|\left(g_{1}^{n} \cdot \nabla g_{1}^{n}\right)_{H F}\right\|_{\widetilde{L}^{1}\left(B_{p_{1}, 1}^{\frac{N}{p_{1}}-1}\right)} \leq C\left\|g_{1}^{n}\right\|_{\widetilde{L}^{\infty}\left(\widetilde{B}_{2, p_{1}, 1}^{\frac{N}{2}-1, \frac{N}{p_{1}}-1}\right)}\left\|\nabla g_{1}^{n}\right\|_{\widetilde{L}^{1}\left(\widetilde{B}_{2, p, 1}^{\frac{N}{2}, \frac{N}{p}}\right)}
$$

We can treat similarly the terms $g_{1}^{n} \cdot \nabla h_{1}^{n}$ and $h_{1}^{n} \cdot \nabla g_{1}^{n}$. We have finally under the conditions (4.17), (4.18), (4.19) and (4.20):

$$
\left\|\left(u^{n} \cdot \nabla u^{n}\right)_{H F}\right\|_{\widetilde{L}^{1}\left(B_{p_{1}, 1}^{\frac{N}{p_{1}}-1}+B_{p, 1}^{\frac{N}{p}}\right)} \leq C\left\|u^{n}\right\|_{E}^{2} .
$$

We finish with the following term where $h$ is a regular function such that $h(0)=0$ :

$$
\begin{aligned}
& \left\|\left[\nabla(\Delta)^{-1}\left(P^{\prime}\left(\rho^{n}\right) \operatorname{div}\left(\rho^{n} u^{n}\right)\right)\right]_{H F}\right\|_{\widetilde{L}^{1}\left(B_{p_{1}, 1}^{\frac{N}{p_{1}}-1}+B_{p, 1}^{\frac{N}{p}}\right)} \\
& \leq\left\|\left[\nabla(\Delta)^{-1}\left(f\left(q^{n}\right) \operatorname{div}\left(q^{n} u^{n}\right)\right)\right]_{H F}\right\|_{\widetilde{L}^{1}\left(B_{p_{1}, 1}^{\frac{N}{p_{1}}-1}+B_{p, 1}^{\frac{N}{p}}\right)} \\
& +\left\|\left[\nabla(\Delta)^{-1}\left(\operatorname{div}\left(q^{n} u^{n}\right)\right)\right]_{H F}\right\|_{\widetilde{L}^{1}\left(B_{p_{1}, 1}^{\frac{N}{p_{1}}-1}+B_{p, 1}^{\frac{N}{p}}\right)}+\left\|\left[\nabla(\Delta)^{-1} \operatorname{div}\left(u^{n}\right)\right]_{H F}\right\|_{\widetilde{L}^{1}\left(B_{p_{1}, 1}^{\frac{N}{p_{1}}-1}+B_{p, 1}^{\frac{N}{p}}\right)}, \\
& \leq C\left\|\operatorname{div} u^{n}\right\|_{\widetilde{L}^{1}\left(\widetilde{B}_{2, p, 1}^{\frac{N}{2}, \frac{N}{p}+1}\right)}\left(\frac{1}{2^{l_{0}}}+\left\|q^{n}\right\|_{\widetilde{L}^{\infty}\left(\widetilde{B}_{2, p, 1}^{\frac{N}{2}, \frac{N}{p}}\right)}+\left\|q^{n}\right\|_{\widetilde{L}^{\infty}\left(\widetilde{B}_{2, p, 1}^{\frac{N}{2}, \frac{N}{p}}\right)}^{2}\right) .
\end{aligned}
$$


In the last inequality we just take in account the fact that $\left[\nabla(\Delta)^{-1} \operatorname{div}\left(u^{n}\right)\right]_{H F}$ is in $\widetilde{L}^{1}\left(B_{p, 1}^{\frac{N}{p}+1}\right)$, so we deduce the following inequality:

$$
\left\|\left[\nabla(\Delta)^{-1} \operatorname{div}\left(u^{n}\right)\right]_{H F}\right\|_{\widetilde{L}^{1}\left(B_{p_{1}, 1}^{\frac{N}{p_{1}}-1}+B_{p, 1}^{\left.\frac{N}{p}\right)}\right.} \leq \frac{1}{2^{l_{0}}}\left\|\operatorname{div} u^{n}\right\|_{\widetilde{L}^{1}\left(\widetilde{B}_{2, p, 1}^{\frac{N}{2}, \frac{N}{p}+1}\right)} .
$$

In the sequel we will need to assume that $l_{0}$ is big enough to assure some bootstrap estimates. We have now to treat the case of low frequencies, in particular estimating $\left\|\left(F^{n}\right)_{B F}\right\|_{\widetilde{L}^{1}\left(B_{2,1}^{\frac{N}{2}-1}\right)}$ and $\left\|\left(G^{n}\right)_{B F}\right\|_{\widetilde{L}^{1}\left(B_{2,1}^{\frac{N}{2}-1}\right)}$. We begin with $\left\|\left(F^{n}\right)_{B F}\right\|_{\widetilde{L}^{1}\left(B_{2,1}^{\frac{N}{2}-1}\right)}$. We have then according proposition 6.8 if $p<\max (4,2 N)$ :

$$
\begin{gathered}
\left\|\left(q^{n} \operatorname{div} u^{n}\right)_{B F}\right\|_{\widetilde{L}^{1}\left(B_{2,1}^{\frac{N}{2}-1,}\right)} \leq\left\|T_{q^{n}}\left(\operatorname{div} u^{n}\right)\right\|_{\widetilde{L}^{1}\left(\widetilde{B}_{2, p, 1}^{\frac{N}{2}-1, \frac{N}{p}-1}\right)}+\left\|T_{\operatorname{div} u^{n}} q^{n}\right\|_{\widetilde{L}^{1}\left(\widetilde{B}_{2, p, 1}^{\frac{N}{2}-1, \frac{N}{p}}\right)} \\
+\left\|\left(R\left(q^{n}, \operatorname{div} u^{n}\right)\right)_{B F}\right\|_{\widetilde{L}^{1}\left(B_{2,1}^{\frac{N}{2}-1}\right)}, \\
\leq C\left(\left\|q^{n}\right\|_{\widetilde{L}^{2}\left(\widetilde{B}_{2, p, 1}^{\left.\frac{N}{2}, \frac{N}{p}\right)}\right.}\left\|u^{n}\right\|_{\widetilde{L}^{2}\left(\widetilde{B}_{2, p, 1}^{\left.\frac{N}{2}, \frac{N}{p}\right)}\right.}+\left\|q^{n}\right\|_{\widetilde{L}^{\infty}\left(\widetilde{B}_{2, p, 1}^{\left.\frac{N}{2}-1, \frac{N}{p}\right)}\right.}\left\|u^{n}\right\|_{\widetilde{L}^{1}\left(\widetilde{B}_{2, p, 1}^{\frac{N}{2}+1, \frac{N}{p}+1}\right)}\right) .
\end{gathered}
$$

Here the only difficulty is to treat the term $R\left(q^{n}, \operatorname{div} u^{n}\right)$ when $N=2$, we need in this case of the previous condition:

$$
p<\max (4,2 N)
$$

and:

$$
\left\|\left(R\left(q^{n}, \operatorname{div} u^{n}\right)\right)_{B F}\right\|_{\widetilde{L}^{1}\left(B_{2,1}^{\frac{N}{2}-1}\right)} \leq C\left\|q^{n}\right\|_{\widetilde{L}^{2}\left(\widetilde{B}_{2, p, 1}^{\left.\frac{N}{2}, \frac{N}{p}\right)}\right.}\left\|u^{n}\right\|_{\widetilde{L}^{2}\left(\widetilde{B}_{2, p, 1}^{\frac{N}{2}, \frac{N}{p}}\right)} \cdot
$$

We now want to estimate $\left\|G^{n}\right\|_{\widetilde{L}^{1}\left(\widetilde{B}_{2, p_{1}, 1}^{\frac{N}{2}-1, \frac{N}{p_{1}}-1}+\widetilde{B}_{2, p, 1}^{\left.\frac{N}{2}-1, \frac{N}{p}\right)}\right.}$, we begin with the following term $\left\|\left(\frac{q^{n}}{1+q^{n}} \mathcal{A} u^{n}\right)_{H F}\right\|_{\widetilde{L}^{1}\left(B_{2,1}^{\frac{N}{2}-1}\right)}$. The main difficulty corresponds to treat $T_{\mathcal{A} u^{n}} \frac{q^{n}}{1+q^{n}}$ and $R\left(\frac{q^{n}}{1+q^{n}}, \mathcal{A} u^{n}\right)$. We have by using proposition 6.8 if: $\frac{1}{2} \leq \frac{2}{p}, N-1>0,2 \frac{N}{p}-1>0$ (we recall here that $\left.\widetilde{L}^{\infty}\left(\widetilde{B}_{2, p, 1}^{\frac{N}{2}, \frac{N}{p}}\right) \hookrightarrow \widetilde{L}^{\infty}\left(\widetilde{B}_{2, p, 1}^{\frac{N}{2}-1, \frac{N}{p}}\right)\right)$ :

$$
\left\|\left(R\left(\frac{q^{n}}{1+q^{n}}, \mathcal{A} u^{n}\right)\right)_{B F}\right\|_{\widetilde{L}^{1}\left(B_{2,1}^{\frac{N}{2}-1}\right)} \leq C\left\|\frac{q^{n}}{1+q^{n}}\right\|_{\widetilde{L}^{\infty}\left(\widetilde{B}_{2, p, 1}^{\left.\frac{N}{2}, \frac{N}{p}\right)}\right.}\left\|\mathcal{A} u^{n}\right\|_{\widetilde{L}^{1}\left(\widetilde{B}_{2, p, 1}^{\frac{N}{2}-1, \frac{N}{p}-1}\right)} .
$$

We need then of the following conditions:

$$
p<\max (4,2 N) .
$$

Next we have according proposition 6.8 with $\lambda=\lambda^{\prime}=+\infty$ :

$$
\begin{aligned}
& \left\|\left(T_{\mathcal{A} u^{n}} \frac{q^{n}}{1+q^{n}}\right)_{B F}\right\|_{\widetilde{L}^{1}\left(B_{2,1}^{\frac{N}{2}-1}\right)} \leq\left\|T_{\mathcal{A} u^{n}} \frac{q^{n}}{1+q^{n}}\right\|_{\widetilde{L}^{1}\left(\widetilde{B}_{2, p, 1}^{\frac{N}{2}-1, \frac{N}{p}-1}\right)}, \\
& \leq C\left\|\frac{q^{n}}{1+q^{n}}\right\|_{\widetilde{L}^{\infty}\left(\widetilde{B}_{2, p, 1}^{\frac{N}{2}, \frac{N}{p}}\right)}\left\|\mathcal{A} u^{n}\right\|_{\widetilde{L}^{1}\left(\widetilde{B}_{2, p, 1}^{\frac{N}{2}-1, \frac{N}{p}-1}\right)}
\end{aligned}
$$

Finally we have:

$$
\left\|\left(K\left(q^{n}\right) \nabla q^{n}\right)_{B F}\right\|_{\widetilde{L}^{1}\left(B_{2,1}^{\frac{N}{2}-1}\right)} \leq\left\|q^{n}\right\|_{\widetilde{L}^{2}\left(\widetilde{B}_{2, p, 1}^{\left.\frac{N}{2}, \frac{N}{p}\right)}\right.}
$$


To finish, it stays in low frequencies the terms: $T_{\nabla u^{n}} u^{n}$ and $R\left(u^{n}, \nabla u^{n}\right)$. We have then by proposition 6.8 if $p<\max (4,2 N)$ :

$$
\left\|\left(R\left(h_{1}^{n}, \nabla h_{1}^{n}\right)\right)_{B F}\right\|_{\widetilde{L}^{1}\left(B_{2,1}^{\frac{N}{2}-1}\right)} \leq C\left\|h_{1}^{n}\right\|_{\widetilde{L}^{\infty}\left(\widetilde{B}_{2, p, 1}^{\frac{N}{2}-1, \frac{N}{p}-1}\right)}\left\|\nabla h_{1}^{n}\right\|_{\widetilde{L}^{1}\left(\widetilde{B}_{2, p, 1}^{\frac{N}{2}, \frac{N}{p}}\right)} .
$$

We have seen that we need again of condition (4.21).

We want now to treat $\left(R\left(g_{1}^{n}, \nabla g_{1}^{n}\right)\right)_{B F}$, we have then by proposition 6.8 if $\frac{N}{p_{1}}+\frac{N}{p}-1>0$ and $\frac{1}{2} \leq \frac{1}{p}+\frac{1}{p_{1}}$

$$
\left\|\left(R\left(g_{1}^{n}, \nabla g_{1}^{n}\right)\right)_{B F}\right\|_{\widetilde{L}^{1}\left(B_{2,1}^{\frac{N}{2}-1}\right)} \leq C\left\|g_{1}^{n}\right\|_{\widetilde{L}^{\infty}\left(\widetilde{B}_{2, p_{1}, 1}^{\frac{N}{2}-1, \frac{N}{p_{1}}-1}\right)}\left\|\nabla g_{1}^{n}\right\|_{\widetilde{L}^{1}\left(\widetilde{B}_{2, p, 1}^{\frac{N}{2}, \frac{N}{p}}\right)} .
$$

We have seen that we need the following conditions:

$$
\frac{1}{2} \leq \frac{1}{p}+\frac{1}{p_{1}} \text { and } \frac{N}{p_{1}}+\frac{N}{p}-1>0 .
$$

Next we have by proposition 6.8 :

$$
\left\|T_{\nabla h_{1}^{n}} h_{1}^{n}\right\|_{\widetilde{L}^{1}\left(\widetilde{B}_{2, p, 1}^{\frac{N}{2}-1, \frac{N}{p}}\right)} \leq C\left\|h_{1}^{n}\right\|_{\widetilde{L}^{\infty}\left(\widetilde{B}_{2, p, 1}^{\frac{N}{2}-1, \frac{N}{p}}\right)}\left\|\nabla h_{1}^{n}\right\|_{\widetilde{L}^{1}\left(\widetilde{B}_{2, p, 1}^{\frac{N}{2}, \frac{N}{p}}\right)}
$$

and

$$
\left\|T_{\nabla g_{1}^{n}} g_{1}^{n}\right\|_{\widetilde{L}^{1}\left(\widetilde{B}_{2, p, 1}^{\left.\frac{N}{2}-1, \frac{N}{p}\right)}\right.} \leq C\left\|g_{1}^{n}\right\|_{\widetilde{L}^{\infty}\left(\widetilde{B}_{2, p_{1}, 1}^{\frac{N}{2}-1, \frac{N}{p_{1}}-1}\right)}\left\|\nabla g_{1}^{n}\right\|_{\widetilde{L}^{1}\left(\widetilde{B}_{2, p, 1}^{\left.\frac{N}{2}, \frac{N}{p}\right)}\right.} .
$$

We proceed similarly to control $T_{u^{n}} \nabla u^{n}$. Therefore the above inequalities with conditions (4.17), (4.18), (4.19), (4.20), (4.21), (4.22) and (4.23) imply that for all $t \in\left[0, T_{n}\right]$ we have if $l_{0}$ is big enough :

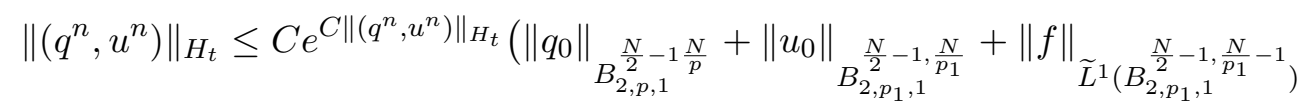

$$
\begin{aligned}
& \left.+\left\|\left(q^{n}, u^{n}\right)\right\|_{H_{t}}^{2}\right) .
\end{aligned}
$$

From a standard bootstrap argument, it is now easy to conclude that there exists a positive constant $c$ such that if the data has been chosen so small as to satisfy:

$$
\left\|q_{0}\right\|_{B_{2, p, 1}^{\frac{N}{2}-1 \frac{N}{p}}}+\left\|u_{0}\right\|_{B_{2, p_{1}, 1}^{\frac{N}{2}-1, \frac{N}{p_{1}}}}+\|f\|_{\widetilde{L}^{1}\left(B_{2, p_{1}, 1}^{\frac{N}{2}-1, \frac{N}{p_{1}}-1}\right)} \leq c .
$$

then $T_{n}=+\infty$. Furthermore it exists $C>0$ such that for all $t \in \mathbb{R}$ :

$$
\left\|\left(q^{n}, u^{n}\right)\right\|_{H_{t}} \leq C, \quad \forall t \in \mathbb{R}
$$

\section{Compactness arguments}

Let us first focus on the convergence of $\left(q^{n}\right)_{n \in \mathbb{N}}$. We claim that, up to extraction, $\left(q^{n}\right)_{n \in \mathbb{N}}$ converges in the distributional sense to some function $q$ such that:

$$
q \in \widetilde{L}^{\infty}\left(\widetilde{B}_{2, p, 1}^{\frac{N}{2}-1, \frac{N}{p}}\right) \cap \widetilde{L}^{1}\left(\widetilde{B}_{2, p, 1}^{\frac{N}{2}+1, \frac{N}{p}}\right) .
$$


The proof is based on Ascoli's theorem and compact embedding for Besov spaces. As similar arguments have been employed in [13] or [17], we only give the outlines of the proof. We may write that:

$$
\partial_{t} q^{n}=-u^{n} \cdot \nabla q^{n}-\left(1+q^{n}\right) \operatorname{div} u^{n} .
$$

Since $\left(u^{n}\right)_{n \in \mathbb{N}}$ is uniformly bounded in $\widetilde{L}^{2}\left(\widetilde{B}_{2, p, 1}^{\frac{N}{2}, \frac{N}{p}+1}+\widetilde{B}_{2, p_{1}, 1}^{\frac{N}{2}, \frac{N}{1}}\right)$ and $q^{n} \in \widetilde{L}^{\infty}\left(\widetilde{B}_{2, p, 1}^{\frac{N}{2}-1, \frac{N}{p}}\right)$, we have $\left(1+q^{n}\right) \operatorname{div} u^{n}$ which is bounded in $\widetilde{L}^{2}\left(\widetilde{B}_{2, p, 1}^{\frac{N}{2}-1, \frac{N}{p}}+\widetilde{B}_{2, p_{1}, 1}^{\frac{N}{2}-1, \frac{N}{p_{1}}-1}\right)$ with the conditions between $p$ and $p_{1}$ in theorem 1.1. Similarly $u^{n} \cdot \nabla q^{n}$ is bounded in $\widetilde{L}_{T}^{2}\left(\widetilde{B}_{2, p, 1}^{\frac{N}{2}-1, \frac{N}{p}}+\widetilde{B}_{2, p_{1}, 1}^{\frac{N}{2}-1, \frac{N}{p_{1}}-1}\right)$. Finally as $p \geq p_{1}$, we have proved that $\partial_{t} q^{n}$ is bounded in $\widetilde{L}_{T}^{2}\left(\widetilde{B}_{2, p, 1}^{\frac{N}{2}-1, \frac{N}{p}-1}\right)$, it means that $\left(q^{n}\right)_{n \in \mathbb{N}}$ seen as a sequence of $\widetilde{B}_{2, p, 1}^{\frac{N}{2}-1, \frac{N}{p}-1}$ valued functions is locally equicontinuous in $\mathbb{R}^{+}$. In addition $\left(q^{n}\right)_{n \in \mathbb{N}}$ is bounded in $C\left(\mathbb{R}^{+}, \widetilde{B}_{2, p, 1}^{\frac{N}{2}-1, \frac{N}{p}-1} \cap \widetilde{B}_{2, p, 1}^{\frac{N}{2}, \frac{N}{p}}\right)$. As the embed$\operatorname{ding} \widetilde{B}_{2, p, 1}^{\frac{N}{2}-1, \frac{N}{p}-1} \cap \widetilde{B}_{2, p, 1}^{\frac{N}{2}, \frac{N}{p}}$ is locally compact (see [3], Chap2), one can thus conclude by means of Ascoli's theorem and Cantor diagonal extraction process that there exists some distribution $q$ such that up to an omitted extraction $\left(\psi q^{n}\right)_{n \in \mathbb{N}}$ converges to $\psi q$ in $C\left(\mathbb{R}^{+}, \widetilde{B}_{2, p, 1}^{\frac{N}{2}-1, \frac{N}{p}-1}\right)$ for all smooth $\psi$ with compact support in $\mathbb{R}^{+} \times \mathbb{R}^{N}$. Then by using the so-called Fatou property for the Besov spaces, one can conclude that (4.24) is satisfied. (the reader may consult [3], Chap 10 too). By proceeding similarly, we can prove that up to extraction, $\left(u^{n}\right)_{n \in \mathbb{N}}$ converges in the distributional sense to some function $u$ such that:

$$
u \in \widetilde{L}^{\infty}\left(\widetilde{B}_{2, p_{1}, 1}^{\frac{N}{2}-1, \frac{N}{p_{1}}-1}+\widetilde{B}_{2, p_{1}, 1}^{\frac{N}{2}-1, \frac{N}{p}}\right) \cap \widetilde{L}^{1}\left(\widetilde{B}_{2, p, 1}^{\frac{N}{2}+1, \frac{N}{p}+1}\right) .
$$

In order to complete the proof of the existence part of theorem 1.1, it is only a matter of checking the continuity properties with respect to time, namely that:

$$
q \in \widetilde{C}\left(\mathbb{R}^{+}, \widetilde{B}_{2, p, 1}^{\frac{N}{2}-1, \frac{N}{p}}\right) \text { and } u \in \widetilde{C}\left(\mathbb{R}^{+}, \widetilde{B}_{2, p, 1}^{\frac{N}{2}-1, \frac{N}{p}}+\widetilde{B}_{2, p_{1}, 1}^{\frac{N}{2}-1, \frac{N}{p_{1}}-1}\right) .
$$

As regards $q$, it suffices to notice that, according to (4.24), (4.25) and to the product laws in the Besov spaces, we have:

$$
\partial_{t} q+u \cdot q=-(1+q) \operatorname{div} u \in \widetilde{L}^{1}\left(\widetilde{B}_{2, p, 1}^{\frac{N}{2}, \frac{N}{p}}\right) .
$$

As $q_{0} \in \widetilde{B}_{2, p, 1}^{\frac{N}{2}, \frac{N}{p}}$, classical results for the transport equation (see [3], Chap 3) ensure that $q \in \widetilde{C}\left(\mathbb{R}^{+}, \widetilde{B}_{2, p, 1}^{\frac{N}{2}, \frac{N}{p}}\right)$. And as previously, we have shown that $q \in \widetilde{C}\left(\mathbb{R}^{+}, \widetilde{B}_{2, p, 1}^{\frac{N}{2}-1, \frac{N}{p}-1}\right)$, it means clearly that $q \in \widetilde{C}\left(\mathbb{R}^{+}, \widetilde{B}_{2, p, 1}^{\frac{N}{2}-1, \frac{N}{p}}\right)$.

For getting the continuity result for $u$, one may similarly use the properties of the heat equation on $v_{1}$ in high frequncies and on $u$ in low frequencies.

\section{The proof of the uniqueness}

In the case $\frac{2}{N} \leq \frac{1}{p}+\frac{1}{p_{1}}$, the uniqueness has been established in [13, 21]. 


\section{$5 \quad$ Proof of the theorems 1.2 and 1.3}

\subsection{Proof of theorem 1.2}

We want here to avoid the condition $p<\max (4,2 N)$. For simplicity we will treat only the case $N=3$. This condition appears when we want to treat the terms of remainder in low frequencies. For resolving this problem as in the paper of F. Charve and R. Danchin in [7], we need an additionnal condition in high frequencies on $q_{0}$ and $u_{0}$.

We want then to follow the same strategy as in the proof of theorem 1.1. It means that we use the same standard scheme which consists in the construction of approximate solutions, some uniform bounds and results of compactness. We will use the same notations as in proof of the theorem 1.1. We just want to treat the non linear term where appears the condition $p<\max (2 N, 4)$ in an other way by using the additional hypothesis that we have on $\left(q_{0}, u_{0}\right) \in \widetilde{B}_{2, \infty}^{0,1} \times B_{2, \infty}^{0}$. We will work with the same space as in the proof of the theorem 1.1 except that we expect additional regularity on $\left(q^{n}, u^{n}\right)$ in $E^{\prime}$ with:

$$
E_{1}^{\prime}=\left(\widetilde{L}^{\infty}\left(\widetilde{B}_{2, \infty}^{0,1}\right) \cap \widetilde{L}^{1}\left(\widetilde{B}_{2, \infty}^{2,1}\right)\right) \times\left(\widetilde{L}^{\infty}\left(\widetilde{B}_{2, \infty}^{0}\right) \cap \widetilde{L}^{1}\left(\widetilde{B}_{2, \infty}^{2}\right)\right) .
$$

Here $\left(q^{n}, u^{n}\right)_{n \in \mathbb{N}}$ is the solution of the following system:

$$
\left\{\begin{array}{l}
\partial_{t} q^{n}+u^{n} \cdot \nabla q^{n}+\operatorname{div} u^{n}=F^{n} \\
\partial_{t} u^{n}+u^{n} \cdot \nabla u^{n}-\mathcal{A} u^{n}+P^{\prime}(1) \nabla q^{n}=G^{n}+f^{n} \\
\left(q^{n}, u^{n}\right)_{/ t=0}=\left(q_{0}^{n}, u_{0}^{n}\right),
\end{array}\right.
$$

which verifies proposition 4 in [7] with:

$$
\begin{aligned}
& F^{n}=-q^{n} \operatorname{div} u^{n}, \\
& G^{n}=-\frac{q^{n}}{1+q^{n}} \mathcal{A} u^{n}+\left(P^{\prime}(1)-P^{\prime}\left(1+q^{n}\right)\right) \nabla q^{n} .
\end{aligned}
$$

We apply exactly the same proof than for theorem 1.1, however we have to complete the uniform bounds by showing that $\left(q^{n}, u^{n}\right)$ is uniformly bounded in $H^{\prime} \cap E_{1}^{\prime}$, moreover we have to treat differently the term in low frequencies where appears the conditions $p<\max (4,2 N)$ and $\frac{1}{2} \leq \frac{1}{p}+\frac{1}{p_{1}}$ by using the fact that $\left(q^{n}, u^{n}\right)$ in $E_{1}^{\prime}$.

We begin with treating the term $\left\|F^{n}\right\|_{\widetilde{L}^{1}\left(B_{2, \infty}^{0,1}\right)}$ and $\left\|G^{n}\right\|_{\widetilde{L}^{1}\left(B_{2, \infty}^{0}\right)}$ by using properties of continuity on the paraproduct and proposition 4 of [7]. We have then:

$$
\left\|T_{q^{n}}^{\prime} \operatorname{div} u^{n}\right\|_{\widetilde{L}^{1}\left(B_{2, \infty}^{1}\right)} \leq\left\|q^{n}\right\|_{L^{\infty}\left(L^{\infty}\right)}\left\|\operatorname{div} u^{n}\right\|_{\widetilde{L}^{1}\left(B_{2, \infty}^{1}\right)} .
$$

Similarly:

$$
\left\|T_{q^{n}}^{\prime} \operatorname{div} u^{n}\right\|_{\widetilde{L}^{1}\left(B_{2, \infty}^{0}\right)} \leq\left\|q^{n}\right\|_{L^{2}\left(L^{\infty}\right)}\left\|\operatorname{div} u^{n}\right\|_{\widetilde{L}^{2}\left(B_{2, \infty}^{0}\right)} .
$$

Next we have:

$$
\|K(q) \nabla q\|_{\widetilde{L}^{1}\left(B_{2, \infty}^{0}\right)} \leq\|q\|_{L^{2}\left(L^{\infty}\right)}\|K(q)\|_{\widetilde{L}^{2}\left(B_{2, \infty}^{1}\right)} .
$$

For the term $\left(\frac{1}{\rho}-\frac{1}{\bar{\rho}}\right) \Delta u=J(q) \Delta u$ with $J$ regular and $J(0)=0$, we have:

$$
\begin{aligned}
& \left\|T_{J(q)} \Delta u\right\|_{\widetilde{L}^{1}\left(B_{2, \infty}^{0}\right)} \leq\|J(q)\|_{L^{\infty}\left(L^{\infty}\right)}\|\Delta u\|_{\widetilde{L}^{1}\left(B_{2, \infty}^{0}\right)}, \\
& \left\|T_{\Delta u} J(q)\right\|_{\widetilde{L}^{1}\left(B_{2, \infty}^{0}\right)} \leq\|J(q)\|_{\widetilde{L}^{\infty}\left(B_{2, \infty}^{1}\right)}\|\Delta u\|_{\widetilde{L}^{1}\left(\widetilde{B}_{2, p, \infty}^{\frac{N}{2}-1, \frac{N}{p}-1}\right)} .
\end{aligned}
$$


Concerning the remainder, we have if $p \geq 2$ :

$$
\|R(J(q), \Delta u)\|_{\widetilde{L}^{1}\left(B_{2, \infty}^{0}\right)} \leq\|J(q)\|_{\widetilde{L}^{\infty}\left(B_{2, \infty}^{1}\right)}\|\Delta u\|_{\widetilde{L}^{1}\left(\widetilde{B}_{2, p, \infty}^{\frac{N}{2}-1, \frac{N}{p}-1}\right)} .
$$

We have then obtained:

$$
\|J(q) \Delta u\|_{\widetilde{L}^{1}\left(B_{2, \infty}^{0}\right)} \leq\|q\|_{\widetilde{L}^{\infty}\left(B_{2, \infty}^{1}\right)}\|u\|_{\widetilde{L}^{1}\left(\widetilde{B}_{2, p, \infty}^{\frac{N}{2}+1, \frac{N}{p}+1}\right)}+\|J(q)\|_{L^{\infty}\left(L^{\infty}\right)}\|\Delta u\|_{\widetilde{L}^{1}\left(B_{2, \infty}^{0}\right)} .
$$

It stays now to treat the terms where appears the conditions $p<\max (4,2 N)$ and $\frac{1}{2} \leq$ $\frac{1}{p}+\frac{1}{p_{1}}$ in an other way. As $u^{n} \in \widetilde{L}^{2}\left(\widetilde{B}_{2, p, 1}^{\frac{N}{2}, \frac{N}{p}+1}+\widetilde{B}_{2, p_{1}, 1}^{\frac{N}{2}, \frac{N}{p_{1}}}\right)$, we set $u^{n}=g^{n}+h^{n}$ with $g^{n} \in \widetilde{L}^{2}\left(\widetilde{B}_{2, p, 1}^{\frac{N}{2}, \frac{N}{p}+1}\right)$ and $h^{n} \in \widetilde{L}^{2}\left(\widetilde{B}_{2, p_{1}, 1}^{\frac{N}{2}, \frac{N}{p_{1}}}\right)$. According to proposition 6.8 , we have:

$$
\begin{aligned}
& \left\|\left(R\left(q^{n}, \operatorname{div} g^{n}\right)\right)_{B F}\right\|_{\widetilde{L}^{1}\left(B_{2,1}^{\frac{N}{2}-1}\right)} \leq C\left\|\left(R\left(q^{n}, \operatorname{div} g^{n}\right)\right)_{B F}\right\|_{\widetilde{L}^{1}\left(B_{2,1}^{0}\right)}, \\
& \leq C\left\|q^{n}\right\|_{\widetilde{L}^{2}\left(B_{2, \infty}^{1}\right)}\left\|\operatorname{div} g^{n}\right\|_{\widetilde{L}^{2}\left(\widetilde{B}_{2, p, 1}^{\frac{N}{2}-1, \frac{N}{p}-1}\right)}, \\
& \leq C\left\|q^{n}\right\|_{\widetilde{L}^{2}\left(B_{2, \infty}^{1}\right)}\left\|\operatorname{div} g^{n}\right\|_{\widetilde{L}^{2}\left(\widetilde{B}_{2, p, 1}^{\frac{N}{2}-1, \frac{N}{p}}\right)} \cdot \\
& \left\|\left(R\left(q^{n}, \operatorname{div} h^{n}\right)\right)_{B F}\right\|_{\widetilde{L}^{1}\left(B_{2,1}^{\frac{N}{2}-1,}\right)} \leq C\left\|\left(R\left(q^{n}, \operatorname{div} h^{n}\right)\right)_{B F}\right\|_{\widetilde{L}^{1}\left(B_{2,1}^{0}\right)}, \\
& \leq C\left\|q^{n}\right\|_{\widetilde{L}^{2}\left(\widetilde{B}_{2, \infty}^{1}\right)}\left\|\operatorname{div} h^{n}\right\|_{\widetilde{L}^{2}\left(\widetilde{B}_{2, p_{1}, 1}^{\frac{N}{2}-1, \frac{N}{p_{1}}-1}\right)} .
\end{aligned}
$$

Next we have:

$$
\begin{aligned}
\|\left(R\left(u^{n}, \nabla u^{n}\right)\right)_{B F} & \left\|_{\widetilde{L}^{1}\left(B_{2,1}^{\frac{N}{2}-1,}\right)} \leq C\right\|\left(R\left(u^{n}, \nabla u^{n}\right)\right)_{B F} \|_{\widetilde{L}^{1}\left(B_{2,1}^{0}\right)}, \\
& \leq C\left\|u^{n}\right\|_{\widetilde{L}^{\infty}\left(B_{2, \infty}^{0}\right)}\left\|\nabla u^{n}\right\|_{\widetilde{L}^{1}\left(\widetilde{B}_{2, p, 1}^{\frac{N}{2}, \frac{N}{p}}\right)}
\end{aligned}
$$

It stays to control the term $R\left(\frac{q^{n}}{1+q^{n}}, \Delta u^{n}\right)$ in low frequencies:

$$
\begin{aligned}
\left\|\left(R\left(\frac{q^{n}}{1+q^{n}}, \Delta u^{n}\right)\right)_{B F}\right\|_{\widetilde{L}^{1}\left(B_{2,1}^{\frac{N}{2}-1,}\right)} & \leq C\left\|\left(R\left(\frac{q^{n}}{1+q^{n}}, \Delta u^{n}\right)\right)_{B F}\right\|_{\widetilde{L}^{1}\left(L^{2}\right)}, \\
& \leq C\left\|q^{n}\right\|_{\widetilde{L}^{\infty}\left(B_{2, \infty}^{1}\right)}\left\|\Delta u^{n}\right\|_{\widetilde{L}^{1}\left(\widetilde{B}_{2, p, 1}^{\frac{N}{2}-1, \frac{N}{p}-1}\right)} .
\end{aligned}
$$

Therefore the above inequalities imply that for all $t \in\left[0, T_{n}\right]$ we have :

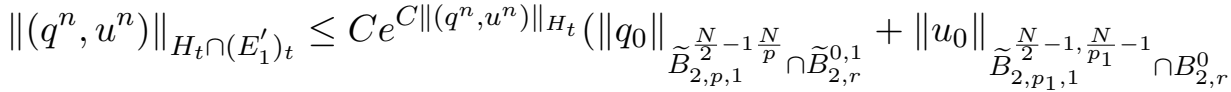

$$
\begin{aligned}
& \left.+\|f\|_{\widetilde{L}^{1}\left(\widetilde{B}_{2, p_{1}, 1}^{\frac{N}{2}-1, \frac{N}{p_{1}}-1} \cap B_{2, r}^{0}\right)}+\left\|\left(q^{n}, u^{n}\right)\right\|_{H_{t} \cap\left(E_{1}^{\prime}\right)_{t}}^{2}\right) .
\end{aligned}
$$

From a standard bootstrap argument, it is now easy to conclude that there exists a positive constant $c$ such that if the data has been chosen so small as to satisfy:

$$
\left\|q_{0}\right\|_{B_{2, p, 1}^{\frac{N}{2}-1 \frac{N}{p}} \cap B_{2, r}^{0,1}}+\left\|u_{0}\right\|_{B_{2, p_{1}, 1}^{\frac{N}{2}-1, \frac{N}{p_{1}}} \cap B_{2, r}^{0}}+\|f\|_{\widetilde{L}^{1}\left(B_{2, p_{1}, 1}^{\frac{N}{2}-1, \frac{N}{p_{1}}-1} \cap B_{2, r}^{0}\right)} \leq c
$$


then $T_{n}=+\infty$. Furthermore it exists $C>0$ such that for all $t \in \mathbb{R}$ :

$$
\left\|\left(q^{n}, u^{n}\right)\right\|_{H_{t} \cap\left(E_{1}^{\prime}\right)_{t}} \leq C, \quad \forall t \in \mathbb{R} .
$$

To conclude we follow the previous proof of the theorem 1.1. Compactness results go along the same lines of the proof of the theorem 1.1 .

\subsection{Proof of theorem $\mathbf{1 . 3}$}

We follow here exactly the lines of the proof of the theorem 1.1 except that we introduce a new effective velocity. Indeed in our case $v$ verifies the following elliptic equation:

$$
\operatorname{div}(\mu(1) D v)+\nabla(\lambda(1) \operatorname{div} v)=\nabla P(\rho)+\operatorname{div}\left(f_{1}(q) D v\right)+\nabla\left(f_{2}(q) \operatorname{div} v\right),
$$

with $f_{1}(q)=\mu(1+q)-\mu(1)$ and $f_{2}(q)=\lambda(1+q)-\lambda(1)$. We can resolve this elliptic equation as $\mu(1) \geq c>0$ and $2 \mu(1)+\lambda(1) \geq c>0$, indeed in our case we work away from the vacuum. To do this we have to use the estimates on the Lamé operator of the appendix in [20]. More precisely we have as $q \in \widetilde{L}^{\infty}\left(\widetilde{B}_{2, p, 1}^{\frac{N}{2}-1, \frac{N}{p}}\right)$ for $r \geq 1, p, q \geq 1$ and $\left|s_{1}\right|<\frac{N}{2},\left|s_{2}\right|<\frac{N}{p}$ :

$$
\|v\|_{\widetilde{L}^{r}\left(\widetilde{B}_{p, q, 1}^{s_{1}, s_{2}}\right)} \leq C\|q\|_{\widetilde{L}^{r}\left(\widetilde{B}_{p, q, 1}^{s_{1}-1, s_{2}-1}\right)}
$$

Indeed as $q$ is small, the terms of remainder with $f_{1}(q)$ and $f_{2}(q)$ are easy to treat. It means that as in the proof of theorem 1.1, $v$ is one derivative more regular than $q$ in high frequencies and that we can estimate $v$ in function of $q$. Moreover we have $\partial_{t} v$ which verifies the following elliptic equation:

$$
\operatorname{div}\left(\mu(\rho) D \partial_{t} v\right)+\nabla\left(\lambda(\rho) \operatorname{div} \partial_{t} v\right)=\nabla \partial_{t} P(\rho)-\operatorname{div}\left(\partial_{t} \mu(\rho) D v\right)-\nabla\left(\partial_{t} \lambda(\rho) \operatorname{div} v\right) .
$$

We can in a similar way getting estimates on $\partial_{t} v$ in function of $q$ and $u$. The rest of the proof is exactly similar to the proof of the theorem 1.1 and is nothing than tedious verifications. It is left to the reader.

\section{Appendix}

This section is devoted to the proof of paraproduct estimates which have been used in section 2 and 3. They are based on paradifferential calculus, a tool introduced by J.M. Bony in [4]. The basic idea of paradifferentiel calculus is that any product of two distributions $u$ and $v$ can be formally decomposed into:

$$
u v=T_{u} v+T_{v} u+R(u, v)=T_{u} v+T_{v}^{\prime} u
$$

where the paraproduct operator is defined by $T_{u} v=\sum_{q} S_{q-1} u \Delta_{q} v$, the remainder operator $R$, by $R(u, v)=\sum_{q} \Delta_{q} u\left(\Delta_{q-1} v+\Delta_{q} v+\Delta_{q+1} v\right)$ and $T_{v}^{\prime} u=T_{v} u+R(u, v)$.

Proposition 6.8 Let $p_{1}, p_{2}, p_{3}, p_{4} \in[1,+\infty],\left(s_{1}, s_{2}, s_{3}, s_{4}\right) \in \mathbb{R}^{4}$ and $(p, q) \in[1,+\infty]^{2}$, we have then the following inequalities: 
- If $\frac{1}{p} \leq \frac{1}{p_{2}}+\frac{1}{\lambda} \leq 1, \frac{1}{q} \leq \frac{1}{p_{4}}+\frac{1}{\lambda^{\prime}} \leq 1$ with $\left(\lambda, \lambda^{\prime}\right) \in[1,+\infty]^{2}$ and $p_{1} \leq \lambda^{\prime}, p_{1} \leq \lambda$, $p_{3} \leq \lambda^{\prime}$ then:

$$
\left\|T_{u} v\right\|_{\widetilde{B}_{p, q, r}^{s_{1}+s_{2}+\frac{N}{p}-\frac{N}{p_{1}}-\frac{N}{p_{2}}, s_{3}+s_{4}+\frac{N}{q}-\frac{N}{p_{3}}-\frac{N}{p_{4}}}} \lesssim\|u\|_{\widetilde{B}_{p_{1}, p_{3}, 1}^{s_{1}, s_{3}}}\|v\|_{\widetilde{B}_{p_{2}, p_{4}, r}^{s_{2}, s_{4}}},
$$

if $s_{1}+\frac{N}{\lambda^{\prime}} \leq \frac{N}{p_{1}}, s_{1}+\frac{N}{\lambda} \leq \frac{N}{p_{1}}$ and $s_{3}+\frac{N}{\lambda^{\prime}} \leq \frac{N}{p_{3}}$.

- If $\frac{1}{q} \leq \frac{1}{p_{3}}+\frac{1}{p_{4}}$ and $s_{3}+s_{4}+N \inf \left(0,1-\frac{1}{p_{3}}-\frac{1}{p_{4}}\right)>0$ then

$$
\sum_{l \geq 4} 2^{l\left(s_{3}+s_{4}+\frac{N}{q}-\frac{N}{p_{3}}-\frac{N}{p_{4}}\right)}\left\|\Delta_{l} R(u, v)\right\|_{L^{q}} \lesssim\|u\|_{\widetilde{B}_{p_{1}, p_{3}, 1}^{s_{1}, s_{3}}}\|v\|_{\widetilde{B}_{p_{2}, p_{4}, r}^{s_{2}, s_{4}}}
$$

- If $\frac{1}{p} \leq \frac{1}{p_{3}}+\frac{1}{p_{4}} \leq 1, \frac{1}{p} \leq \frac{1}{p_{3}}+\frac{1}{p_{2}} \leq 1, \frac{1}{p} \leq \frac{1}{p_{1}}+\frac{1}{p_{4}} \leq 1, \frac{1}{p} \leq \frac{1}{p_{1}}+\frac{1}{p_{2}} \leq 1$ and $s_{3}+s_{4}>0, s_{3}+s_{2}>0, s_{4}+s_{1}>0, s_{1}+s_{2}>0$ then

$$
\sum_{l \leq 4} 2^{l\left(s_{1}+s_{2}+\frac{N}{p}-\frac{N}{p_{1}}-\frac{N}{p_{2}}\right)}\left\|\Delta_{l} R(u, v)\right\|_{L^{p}} \lesssim\|u\|_{\widetilde{B}_{p_{1}, p_{3}, 1}^{s_{1}, \frac{N}{p_{3}}-\frac{N}{p_{1}}+s_{1}}}\|v\|_{\widetilde{B}_{p_{2}, p_{4}, r}^{s_{2}, \frac{N}{p_{4}}-\frac{N}{p_{2}}+s_{2}}} .
$$

with $s_{3}=\frac{N}{p_{3}}-\frac{N}{p_{1}}+s_{1}$ and $s_{4}=\frac{N}{p_{4}}-\frac{N}{p_{2}}+s_{2}$.

- If $u \in L^{\infty}$, we also have:

$$
\left\|T_{u} v\right\|_{\widetilde{B}_{p, q, r}^{s_{1}, s_{2}}} \lesssim\|u\|_{L^{\infty}}\|v\|_{\widetilde{B}_{p, q, r}^{s_{1}, s_{2}}}
$$

and if $\min \left(s_{1}, s_{2}\right)>0$ then:

$$
\|R(u, v)\|_{\widetilde{B}_{p, q, r}^{s_{1}, s_{2}}} \lesssim\|u\|_{L^{\infty}}\|v\|_{\widetilde{B}_{p, q, r}^{s_{1}, s_{2}}}
$$

Proof: Let us prove (6.27). According to the decomposition of J.-M. Bony [4], we have:

$$
u v=T_{u} v+T_{v} u+R(u, v),
$$

so for all $l>0$ :

$$
\Delta_{l} T_{u} v=\sum_{\left|l-l^{\prime}\right| \leq 3} \Delta_{l}\left(S_{l^{\prime}-1} u \Delta_{l^{\prime}} v\right)
$$

For $\alpha, \beta \in \mathbb{R}$, let us define the following characteristic function on $\mathbb{Z}$

$$
\begin{array}{ll}
\varphi^{\alpha, \beta}=\alpha & \text { if } r \leq 0, \\
\varphi^{\alpha, \beta}=\beta & \text { if } r \geq 1 .
\end{array}
$$

if $\frac{1}{p} \leq \frac{1}{p_{2}}+\frac{1}{\lambda} \leq 1$ and $\frac{1}{q} \leq \frac{1}{p_{4}}+\frac{1}{\lambda^{\prime}} \leq 1$ then

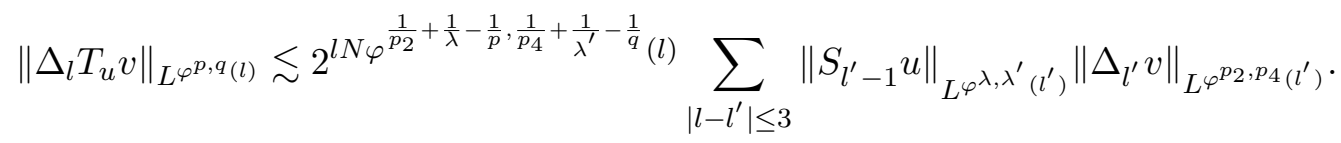


We have by Berstein inequalities and as $p_{1} \leq \lambda^{\prime}, p_{3} \leq \lambda^{\prime}, p_{1} \leq \lambda$ and $s_{1}+\frac{N}{\lambda} \leq \frac{N}{p_{1}}$, $s_{1}+\frac{N}{\lambda^{\prime}} \leq \frac{N}{p_{1}}, s_{3}+\frac{N}{\lambda^{\prime}} \leq \frac{N}{p_{3}}$ :

$$
\begin{aligned}
& \left\|S_{l^{\prime}-1} u\right\|_{L^{\varphi^{\lambda, \lambda^{\prime}}\left(l^{\prime}\right)}} \lesssim \sum_{k \leq l^{\prime}-2} 2^{k\left(\varphi^{\frac{N}{p_{1}}, \frac{N}{p_{3}}}(k)-\varphi^{\frac{N}{\lambda}, \frac{N}{\lambda^{\prime}}}\left(l^{\prime}\right)\right)}\left\|\Delta_{k} u\right\|_{L^{\varphi^{p_{1}, p_{3}(k)}}}, \\
& \lesssim \sum_{k \leq l^{\prime}-2} 2^{k\left(\varphi^{\frac{N}{p_{1}}-s_{1}, \frac{N}{p_{3}}-s_{3}}(k)-\varphi^{\frac{N}{\lambda}, \frac{N}{\lambda^{\prime}}}\left(l^{\prime}\right)\right)} 2^{k \varphi^{s_{1}, s_{3}}(k)}\left\|\Delta_{k} u\right\|_{L^{\varphi^{p_{1}, p_{3}}(k)}},
\end{aligned}
$$

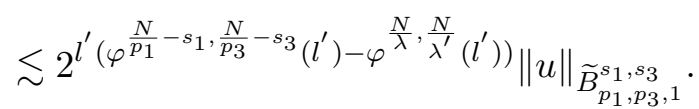

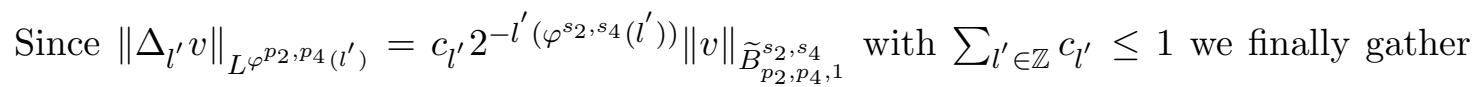
as $l>0$ :

$$
\left\|\Delta_{l} T_{u} v\right\|_{L^{q}} \lesssim c_{l} 2^{l \varphi^{\frac{N}{p_{1}}+\frac{N}{p_{2}}-\frac{N}{p}-s_{1}-s_{2}, \frac{N}{p_{2}}+\frac{N}{p_{4}}-\frac{N}{q}-s_{3}-s_{4}}(l)}\|u\|_{\widetilde{B}_{p_{1}, p_{3}, 1}^{s_{1}, s_{3}}}\|v\|_{\widetilde{B}_{p_{2}, p_{4}, 1}^{s_{2}, s_{4}}} .
$$

And we obtain (6.27).

Straightforward modification give (6.30). In this case as $\left\|S_{k-1} u\right\|_{L^{\infty}} \leq\|u\|_{L^{\infty}}$ we have:

$$
\left\|\Delta_{l} T_{u} v\right\|_{L^{\varphi^{p, q}(l)}} \lesssim \sum_{\left|l-l^{\prime}\right| \leq 3}\|u\|_{L^{\infty}}\left\|\Delta_{l^{\prime}} v\right\|_{L^{\varphi^{p}}, p_{4}\left(l^{\prime}\right)}
$$

Next we have:

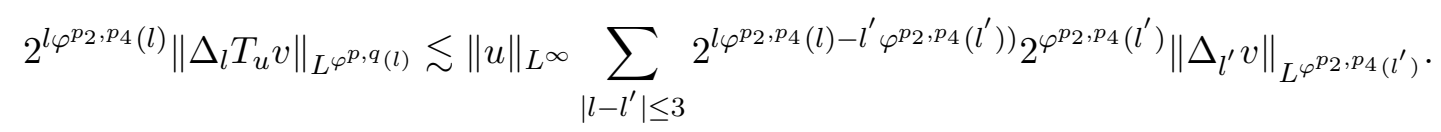

We conclude by convolution.

To prove (6.29), we write:

$$
\Delta_{l} R(u, v)=\sum_{k \geq l-2} \Delta_{l}\left(\Delta_{k} u \widetilde{\Delta}_{k} v\right)
$$

We consider now the case $l>3$. By Bernstein and Hölder inequalities we obtain when $\frac{1}{q} \leq \frac{1}{p_{3}}+\frac{1}{p_{4}} \leq 1$ :

$$
\left\|\Delta_{l} R(u, v)\right\|_{L^{q}} \lesssim 2^{N l\left(\frac{1}{p_{3}}+\frac{1}{p_{4}}-\frac{1}{q}\right)} \sum_{k \geq l-2}\left\|\Delta_{k} u\right\|_{L^{p_{3}}}\left\|\widetilde{\Delta}_{k} v\right\|_{L^{p_{4}}}
$$

Next we have:

$$
\begin{aligned}
2^{l\left(s_{3}+s_{4}+\frac{N}{q}-\frac{N}{p_{3}}-\frac{N}{p_{4}}\right)}\left\|\Delta_{l} R(u, v)\right\|_{L^{q}} & \lesssim \sum_{k \geq l-2} 2^{(l-k)\left(s_{3}+s_{4}\right)} 2^{k s_{3}}\left\|\Delta_{k} u\right\|_{L^{p_{3}}} 2^{k s_{4}}\left\|\widetilde{\Delta}_{k} v\right\|_{L^{p_{4}}}, \\
& \lesssim\left(c_{k}\right) *\left(d_{k^{\prime}}\right),
\end{aligned}
$$


with $c_{k}=1_{[-\infty, 2]}(k) 2^{k\left(s_{3}+s_{4}\right)}$ and $d_{k^{\prime}}=2^{k^{\prime} s_{3}}\left\|\Delta_{k} u\right\|_{L^{p_{3}}} 2^{k^{\prime} s_{4}}\left\|\widetilde{\Delta}_{k} v\right\|_{L^{p_{4}}}$. We conclude by Young inequality as $s_{3}+s_{4}>0$.

We have to treat now the case when $l<0$. We have then as $\frac{1}{p} \leq \frac{1}{p_{3}}+\frac{1}{p_{4}} \leq 1$ and $\frac{1}{p} \leq \frac{1}{p_{1}}+\frac{1}{p_{2}} \leq 1$ :

$$
\begin{aligned}
& \left\|\Delta_{l} R(u, v)\right\|_{L^{p}} \lesssim 2^{N l\left(\frac{1}{p_{3}}+\frac{1}{p_{4}}-\frac{1}{p}\right)} \sum_{k \geq 2}\left\|\Delta_{k} u\right\|_{L^{p_{3}}}\left\|\widetilde{\Delta}_{k} v\right\|_{L^{p_{4}}} \\
& +\sum_{0 \leq k \leq 1,\left|k-k^{\prime}\right| \leq 1}\left\|\Delta_{k} u \Delta_{k^{\prime}} v\right\|_{L^{p}}+2^{N l\left(\frac{1}{p_{1}}+\frac{1}{p_{2}}-\frac{1}{p}\right)} \sum_{l-2 \leq k \leq-1}\left\|\Delta_{k} u\right\|_{L^{p_{1}}}\left\|\widetilde{\Delta}_{k} v\right\|_{L^{p_{2}}} .
\end{aligned}
$$

And by convolution on the middle frequencies:

$$
\begin{aligned}
\left\|\Delta_{l} R(u, v)\right\|_{L^{p}} \lesssim 2^{N l\left(\frac{1}{p_{3}}+\frac{1}{p_{4}}-\frac{1}{p}\right)} \sum_{k \geq 2}\left\|\Delta_{k} u\right\|_{L^{p_{3}}}\left\|\widetilde{\Delta}_{k} v\right\|_{L^{p_{4}}}+\left(2^{l\left(\frac{N}{p_{3}}+\frac{N}{p_{2}}-\frac{N}{p}-s_{3}-s_{2}\right)} c_{l}\right. \\
+2^{l\left(\frac{N}{p_{1}}+\frac{N}{p_{4}}-\frac{N}{p}-s_{1}-s_{4}\right)} c_{l}+2^{N l\left(\frac{1}{p_{1}}+\frac{1}{p_{2}}-\frac{1}{p}\right)} \sum_{l-2 \leq k \leq-1}\left\|\Delta_{k} u\right\|_{L^{p_{1}}}\left\|\widetilde{\Delta}_{k} v\right\|_{L^{p_{2}}},
\end{aligned}
$$

with $c_{l} \in l^{1}(\mathbb{Z})$. Next by convolution we obtain:

$$
\begin{aligned}
\left\|\Delta_{l} R(u, v)\right\|_{L^{p}} \lesssim c_{l}\left(2^{l\left(\frac{N}{p_{3}}+\frac{N}{p_{4}}-\frac{N}{p}-s_{3}-s_{4}\right)}+2^{l\left(\frac{N}{p_{3}}+\frac{N}{p_{2}}-\frac{N}{p}-s_{3}-s_{2}\right)}+2^{l\left(\frac{N}{p_{1}}+\frac{N}{p_{4}}-\frac{N}{p}-s_{1}-s_{4}\right)}\right. \\
\left.+2^{l\left(\frac{N}{p_{1}}+\frac{N}{p_{2}}-\frac{N}{p}-s_{1}-s_{2}\right)}\right)\|u\|_{\widetilde{B}_{p_{1}, p_{3}, 1}^{s_{1}, s_{3}}}\|v\|_{\widetilde{B}_{p_{2}, p_{4}, r}^{s_{2}, s_{4}}} .
\end{aligned}
$$

And we can conclude.

We want prove now the inequality (6.30). We have then:

$$
2^{l \varphi^{s_{1}, s_{2}}(l)}\left\|\Delta_{l} R(u, v)\right\|_{L^{p}} \lesssim \sum_{k \geq l-2} 2^{(l-k) \varphi^{s_{1}, s_{2}}(l)} 2^{k \varphi^{s_{1}, s_{2}}(l)}\left\|\Delta_{k} u\right\|_{L^{\infty}}\left\|\widetilde{\Delta}_{k} v\right\|_{L^{\varphi} p, q(k)},
$$

And we conclude by Young inequality.

\section{References}

[1] H. Abidi and M. Paicu. Équation de Navier-Stokes avec densité et viscosité variables dans l'espace critique. Annales de l'institut Fourier, 57 no. 3 (2007), p. 883-917.

[2] H. Bahouri and J.-Y. Chemin, Équations d'ondes quasilinéaires et estimation de Strichartz, Amer. J. Mathematics. 121 (1999) 1337-1377.

[3] H. Bahouri, J.-Y. Chemin and R. Danchin. Fourier analysis and nonlinear partial differential equations, Grundlehren der mathematischen Wissenschaften, 343, Springer Verlag, 2011.

[4] J.-M. Bony, Calcul symbolique et propagation des singularités pour les équations aux dérivées partielles non linéaires, Annales Scientifiques de l'école Normale Supérieure. 14 (1981) 209-246. 
[5] D. Bresch and B. Desjardins, Existence of global weak solutions for a 2D Viscous shallow water equations and convergence to the quasi-geostrophic model. Comm. Math. Phys., 238(1-2): 211-223, 2003.

[6] D. Bresch and B. Desjardins, Sur un modèle de Saint- Venant visqueux et sa limite quasi-géostrophique. C. R. Math. Acad. Sci. Paris, 335(12):10791084, 2002.

[7] F. Charve and R. Danchin, A global existence result for the compressible NavierStokes equations in the critical $L^{p}$ framework, Archive for Rational Mechanics and Analysis, 198(1), 2010, 233-271.

[8] J.-Y. Chemin, Théorèmes d'unicité pour le système de Navier-Stokes tridimensionnel, J.d'Analyse Math. 77 (1999) 27-50.

[9] J.-Y. Chemin, About Navier-Stokes system, Prépublication du Laboratoire d'Analyse Numérique de Paris 6, R96023 (1996).

[10] J.-Y. Chemin and N. Lerner, Flot de champs de vecteurs non lipschitziens et équations de Navier-Stokes, J.Differential Equations, 121 (1992) 314-328.

[11] Q. Chen, C. Miao and Z. Zhang, Global well-posedness for the compressible NavierStokes equations with the highly oscillating initial velocity, Communications on Pure and Applied Mathematics, Volume 63(2010) 1173-1224.

[12] R. Danchin, Fourier analysis method for PDE's, Preprint, Novembre 2005.

[13] R. Danchin, Local Theory in critical Spaces for Compressible Viscous and HeatConductive Gases, Communication in Partial Differential Equations, 26 (78),11831233, (2001).

[14] R. Danchin, Global existence in critical spaces for compressible Navier-Stokes equations, Inventiones Mathematicae, 141, pages 579-614 (2000).

[15] R. Danchin, Global existence in critical spaces for compressible viscous and heatconductive gases, Archiv for Rational Mechanics and Analysis, 160, pages 1-39 (2001).

[16] R. Danchin, On the uniqueness in critical spaces for compressible Navier-Stokes equations. NoDEA Nonlinear Differentiel Equations Appl, 12(1):111-128, 2005.

[17] R. Danchin, Well-Posedness in critical spaces for barotropic viscous fluids with truly not constant density, Communications in Partial Differential Equations, 32:9,13731397.

[18] B. Haspot, Cauchy problem for Navier-Stokes system with a specific term of capillarity, M3AS, Vol. 20, No. 7 (2010) 1-39.

[19] B. Haspot, Cauchy problem for viscous shallow water equations with a term of capillarity ,Hyperbolic problems: theory, numerics and applications, 625-634, Proc. Sympos. Appl. Math., 67, Part 2, Amer. Math. Soc., Providence, RI, 2009.

[20] B. Haspot, Local well-posedness results for density-dependent incompressible fluids, Arxiv, 0902.1982 (February 2009). 
[21] B. Haspot, Well-posedness in critical spaces for barotropic viscous fluids, Arxiv, (March 2009).

[22] B. Haspot , Regularity of weak solution for compressible barotropic Navier-Stokes equations, Arxiv (Januar 2010) and preprint .

[23] D. Hoff. Global existence for 1D, compressible, isentropic Navier-Stokes equations with large initial data. Trans. Amer. Math. Soc, 303(1), 169-181, 1987.

[24] D. Hoff, Uniqueness of weak solutions of the Navier-Stokes equations of multidimensional, compressible flow, SIAM J. Math. Anal. 37 (6) (2006).

[25] D. Hoff. Discontinuous solutions of the Navier-Stokes equations for multidimensional flows of the heat conducting fluids. Arch. Rational Mech. Anal., 139, (1997), p. 303-354.

[26] D. Hoff. Global solutions of the Navier-Stokes equations for multidimensional compressible flow with discontinuous initial data. J. Differential Equations, 120(1), 215254, 1995.

[27] D. Hoff. Strong convergence to global solutions for multidimensional flows of compressible, viscous fluids with polytropic equations of state and discontinuous initial data. Arch. Rational Mech. Anal., 132(1), 1-14, 1995.

[28] D. Hoff, Dynamics of singularity surfaces for compressible, viscous flows in two space dimensions. Comm. Pure Appl. Math. 55 (2002), no. 11, 13651407.

[29] D. Hoff, Compressible flow in a half-space with Navier boundary conditions. J. Math. Fluid Mech. 7 (2005), no. 3, 315338.

[30] D. Hoff and M. Santos, Lagrangean structure and propagation of singularities in multidimensional compressible flow. Arch. Ration. Mech. Anal. 188 (2008), no. 3, 509543.

[31] D. Hoff and K. Zumbrun. Multi-dimensional diffusion waves for the Navier-Stokes equations of compressible flow, Indiana University Mathematics Journal, 1995, 44, 603-676.

[32] A. V. Kazhikov. The equation of potential flows of a compressible viscous fluid for small Reynolds numbers: existence, uniqueness and stabilization of solutions. Sibirsk. Mat. Zh., 34 (1993), no. 3, p. 70-80.

[33] A. V. Kazhikov and V. V. Shelukhin. Unique global solution with respect to time of initial-boundary value problems for one- dimensional equations of a viscous gas. Prikl. Mat. Meh., 41(2): 282-291, 1977.

[34] P.-L. Lions. Mathematical Topics in Fluid Mechanics, Vol 2, Compressible models, Oxford University Press, (1998).

[35] Akitaka Matsumura and Takaaki Nishida. The initial value problem for the equations of motion of compressible viscous and heat-conductive gases. J. Math. Kyoto Univ., 20(1): 67-104, 1980. 
[36] Akitaka Matsumura and Takaaki Nishida. The initial value problem for the equations of motion of compressible viscous and heat-conductive fluids. Proc. Japan Acad. Ser. A Math. Sci, 55(9):337-342, 1979.

[37] Y. Meyer. Wavelets,paraproducts, and Navier-Stokes equation. In Current developments in mathematics, 1996 (Cambridge, MA), page 105-212. Int. Press, Boston, MA, 1997.

[38] J. Nash. Le problème de Cauchy pour les équations différentielles d'un fluide général. Bull. Soc. Math. France, 90: 487-497, 1962.

[39] T. Runst and W. Sickel: Sobolev spaces of fractional order, Nemytskij operators, and nonlinear partial differential equations. de Gruyter Series in Nonlinear Analysis and Applications, 3. Walter de Gruyter and Co., Berlin (1996)

[40] D. Serre. Solutions faibles globales des équations de Navier-Stokes pour un fluide compressible.Comptes rendus de l'Académie des sciences. Série 1, 303(13): 639-642, 1986.

[41] V. A. Solonnikov. Estimates for solutions of nonstationary Navier-Stokes systems. Zap. Nauchn. Sem. LOMI, 38, (1973), p.153-231; J. Soviet Math. 8, (1977), p. 467-529.

[42] V. Valli and W. Zajaczkowski. Navier-Stokes equations for compressible fluids: global existence and qualitative properties of the solutions in the general case. Commun. Math. Phys., 103, (1986) no 2, p. 259-296. 